\title{
Disjointness preserving operators on normed pre-Riesz spaces: extensions and inverses
}

\author{
Anke Kalauch ${ }^{1}$ - Onno van Gaans ${ }^{2}$ - Feng Zhang ${ }^{2}$ \\ Received: 24 September 2018 / Accepted: 24 May 2019 / Published online: 3 June 2019 \\ (c) The Author(s) 2019
}

\begin{abstract}
We explore inverses of disjointness preserving bijections in infinite dimensional normed pre-Riesz spaces by several methods. As in the case of Banach lattices, our aim is to show that such inverses are disjointness preserving. One method is extension of the operator to the Riesz completion, which works under suitable denseness and continuity conditions. Another method involves a condition on principle bands. Examples illustrate the differences to the Riesz space theory.
\end{abstract}

Keywords Disjointness preserving operator · Extension · Inverse · Pervasive · Pre-Riesz space $\cdot$ Principal band $\cdot$ Riesz completion

Mathematics Subject Classification 47B60 - 46B40

\section{Introduction}

In 1992, Y. A. Abramovich raised a question in the problem section of [6]: if $X, Y$ are vector lattices and $T: X \rightarrow Y$ is a linear disjointness preserving bijection, is $T^{-1}: Y \rightarrow X$ disjointness preserving as well? If $X$ and $Y$ are Banach lattices, the answer is affirmative, see [5]. This initiated research to explore the most general setting in which a similar result is true; see [2] and references therein. It is also observed there that for a disjointness preserving bijection between arbitrary vector lattices the inverse is not disjointness preserving, in general. In [7] it is proved that an order bounded

\footnotetext{
Onno van Gaans

vangaans@math.leidenuniv.nl

Anke Kalauch

anke.kalauch@tu-dresden.de

Feng Zhang

zhangfeng.0631@163.com

1 Institut für Analysis, Fakultät Mathematik, TU Dresden, 01062 Dresden, Germany

2 Mathematical Institute, Leiden University, P.O. Box 9512, 2300 RA Leiden, The Netherlands
} 
disjointness preserving bijection between Archimedean vector lattices has an order bounded disjointness preserving inverse. A result clarifying the role of the underlying spaces is given in [5, Theorem 2.1], which reads as follows.

Theorem 1.1 Let X be a uniformly complete vector lattice, $Y$ a normed vector lattice, and $T: X \rightarrow Y$ an injective and disjointness preserving operator. Then for every $x_{1}, x_{2} \in X$ we have that $T x_{1} \perp T x_{2}$ implies $x_{1} \perp x_{2}$.

In general partially ordered vector spaces there is a natural notion of disjointness, see [9]. In [15], van Haandel develops a theory for pre-Riesz spaces, which are exactly those partially ordered vector spaces that can be order densely embedded into vector lattices. The smallest vector lattice in which a pre-Riesz space can be order densely embedded is called the Riesz completion. In particular, every directed Archimedean partially ordered vector space is a pre-Riesz space. For the theory of pre-Riesz spaces, see also [10]. Abramovich's question extends naturally to the pre-Riesz setting. So far, the problem is only studied in finite dimensions. In [8], it is shown that the inverses of disjointness preserving bijections in finite dimensional Archimedean pre-Riesz spaces are disjointness preserving. The affirmative answer in the finite-dimensional setting motivates to investigate the infinite-dimensional case. The present paper is a first exploration of Abramovich's problem for the infinite-dimensional pre-Riesz space setting. We consider several approaches and examples. Among them, we try to generalize Theorem 1.1 by extending disjointness preserving operators on pre-Riesz spaces to Riesz completions.

The paper is organized as follows. In Sect. 2, we collect some basic terminology and properties. In Sect. 3 we compare the setting of pre-Riesz spaces to the vector lattice setting by means of two examples. In Sect. 4 we show that there may exist a rich set of disjointness preserving operators on a pre-Riesz space by computing all disjointness preserving bijections in a finite dimensional example with a particular polyhedral cone. In the Sects. 5 and 6 we consider generalization of Theorem 1.1. In Sect. 5 we consider more general range spaces, which turns out to be straightforward. Generalizing the domain space $X$ is a more difficult task, as is discussed in Sect. 6 . Our method is to extend disjointness preserving operators on pre-Riesz spaces to their Riesz completions. We study several ways, each of them involving quite strong conditions. Finally, in Sect. 7 we consider a pre-Riesz space generalization of Abramovich and Kitover's analysis of inverses of disjointness preserving operators in [1], which involves a property called property $(\beta)$.

\section{Preliminaries}

We collect some basic terminology; see, e.g., $[3,10]$. Let $X$ be a real vector space and let $K$ be a cone in $X$, that is, $K$ is a wedge $(x, y \in K, \lambda, \mu \geq 0$ imply $\lambda x+\mu y \in K)$ and $K \cap(-K)=\{0\}$. For $x, y \in X$, a partial order in $X$ is induced by

$x \leq y$ whenever $y-x \in K$. 
We call $(X, K)$ then a (partially) ordered vector space. We write $x>0$ whenever $x \in K \backslash\{0\}$. For $M \subseteq X$, we denote

$$
\begin{aligned}
& M^{\mathrm{u}}:=\{x \in X: \text { for all } y \in M \text { one has } x \geq y\} \text { and } \\
& M^{1}:=\{x \in X: \text { for all } y \in M \text { one has } x \leq y\} .
\end{aligned}
$$

The space $X$ has the Riesz decomposition property if for every $x_{1}, x_{2}, z \in K$ with $z \leq x_{1}+x_{2}$ there exist $z_{1}, z_{2} \in K$ such that $z=z_{1}+z_{2}$ with $z_{1} \leq x_{1}$ and $z_{2} \leq x_{2}$.

Definition 2.1 A partially ordered vector space $X$ is called a pre-Riesz space if for every $x, y, z \in X$ such that $\{x+y, x+z\}^{\mathrm{u}} \subseteq\{y, z\}^{\mathrm{u}}$ one has that $x \geq 0$.

Let $X$ be a partially ordered vector space. Recall that $X$ is Archimedean if for every $x, y \in X$ with $n x \leq y$ for all $n \in \mathbb{N}$ one has $x \leq 0$. The space $X$ is called directed if for every $x, y \in X$ there exists $z \in X$ such that $z \geq x$ and $z \geq y$. Every pre-Riesz space is directed and every Archimedean directed ordered vector space is pre-Riesz.

A linear subspace $D$ of $X$ is called order dense in $X$ if for every $x \in X$ we have $x=\inf \{y \in D: y \geq x\}$. Let $Y$ be a partially ordered vector space. A linear map $i: X \rightarrow Y$ is called bipositive if for every $x \in X$ one has $i(x) \geq 0$ if and only if $x \geq 0$. A subspace $D$ of $X$ is called majorizing if for every $x \in X$ there exists $y \in D$ such that $x \leq y$. Clearly, every order dense subspace is majorizing. We say that a subspace $X$ of a vector lattice $Y$ generates $Y$ as a vector lattice if for every $y \in Y$ there exist $a_{1}, \ldots, a_{m}, b_{1}, \ldots, b_{n} \in X$ such that $y=\bigvee_{i=1}^{m} a_{i}-\bigvee_{j=1}^{n} b_{j}$.

Theorem 2.2 [15, Corollaries 4.9-11 and Theorems 3.5, 3.7, 4.13] Let X be a partially ordered vector space. The following statements are equivalent.

(i) $X$ is pre-Riesz.

(ii) There exist a vector lattice $Y$ and a bipositive linear map $i: X \rightarrow Y$ such that $i(X)$ is order dense in $Y$.

(iii) There exist a vector lattice $Y$ and a bipositive linear map $i: X \rightarrow Y$ such that $i(X)$ is order dense in $Y$ and $i(X)$ generates $Y$ as a vector lattice.

A pair $(Y, i)$ as in (ii) is called a vector lattice cover of $X$. A pair $(Y, i)$ as in (iii) is unique up to isomorphism and called the Riesz completion of $X$, denoted by $\left(X^{\rho}, i\right)$. Note that if $X$ is Archimedean, then the classical Dedekind completion $\left(X^{\delta}, j\right)$ of $X$ is a vector lattice cover; for details see [10, Chapter 2].

Let $X$ be a pre-Riesz space. Two elements $x, y \in X$ are called disjoint, denoted by $x \perp y$, if $\{x+y,-x-y\}^{\mathrm{u}}=\{x-y,-x+y\}^{\mathrm{u}}$. The disjoint complement of $M \subseteq X$ is defined by $M^{\mathrm{d}}:=\{y \in X$ : for every $x \in M$ one has $x \perp y\}$.

Theorem 2.3 ([9], Proposition 2.1) Let $X$ be a pre-Riesz space and let $(Y, i)$ be a vector lattice cover of $X$. Then for every $x, y \in X$ we have $x \perp y$ if and only if $i(x) \perp i(y)$.

We will need the following technical observation. If $D$ is a majorizing subspace of a vector lattice $Y$ and $u \in Y$ is such that $u \perp d$ for every $d \in D$, then $u=0$. Indeed, there is $w \in D$ such that $|u| \leq w$. Hence, $|u|=|u| \wedge w=0$, consequently $u=0$. We conclude the following lemma. 
Lemma 2.4 If $X$ is a pre-Riesz space and $u \in X^{\rho}$ is such that $u \perp x$ for every $x \in i(X)$, then $u=0$.

Definition 2.5 Let $X$ be a pre-Riesz space and let $\left(X^{\rho}, i\right)$ be the Riesz completion of $X$. The space $X$ is called fordable if for every $y \in X^{\rho}, y \geq 0$, there is $M \subseteq X$ such that $\{y\}^{\mathrm{d}}=i(M)^{\mathrm{d}}$.

Definition 2.6 A pre-Riesz space $X$ with the Riesz completion $\left(X^{\rho}, i\right)$ is called pervasive if for every $y \in X^{\rho}$ with $0<y$ there exists $x \in X$ such that $0<i(x) \leq y$.

It is known that every pervasive pre-Riesz space is fordable [10, Proposition 4.1.15], whereas the converse is not true, in general, see [10, Example 4.1.19]. If a pre-Riesz space $X$ is pervasive and $(Y, i)$ is any vector lattice cover of $X$, then for every $y \in Y$ with $y>0$ there exists $x \in X$ with $0<i(x) \leq y$, see [10, Proposition 2.8.8].

Let $X$ and $Y$ be pre-Riesz spaces. A linear operator $T: X \rightarrow Y$ is called disjointness preserving if for every $x, y \in X$ with $x \perp y$ we have $T x \perp T y$.

A seminorm $\|\cdot\|$ on an ordered vector space $X$ is called monotone if $\|x\| \leq\|y\|$ for every $x, y \in X$ with $0 \leq x \leq y$. A seminorm $\|\cdot\|$ on a Riesz spae $X$ is a Riesz seminorm if it is monotone and $\||x|\|=\|x\|$ for every $x \in X$. By a normed Riesz space we mean a Riesz space endowed with a Riesz norm. The cone of a normed Riesz space is closed and therefore every normed Riesz space is Archimedean.

For a net $\left(x_{\alpha}\right)_{\alpha \in I}$ in an ordered vector space $(X, K)$ and $x \in X$ we write $x_{\alpha} \uparrow x$ if $\left(x_{\alpha}\right)_{\alpha}$ is increasing and $x$ is the supremum of $\left\{x_{\alpha}: \alpha \in I\right\}$. Similarly, we use the notation $x_{\alpha} \downarrow x$. The net $\left(x_{\alpha}\right)_{\alpha \in I}$ order converges, in short o-converges, to $x \in X$ (denoted by $x_{\alpha} \stackrel{\circ}{\rightarrow} x$ ), if there is a net $\left(y_{\alpha}\right)_{\alpha \in I}$ in $X$ such that $y_{\alpha} \downarrow 0$ and for every $\alpha \in I$ one has $\pm\left(x_{\alpha}-x\right) \leq y_{\alpha}$. A linear operator $T: X \rightarrow Y$ between two ordered vector spaces is said to be order continuous if $T x_{\alpha} \stackrel{\circ}{\rightarrow} 0$ whenever $x_{\alpha} \stackrel{\circ}{\rightarrow} 0$.

For $u \in K$, a sequence $\left(x_{n}\right)_{n}$ in $X$ is said to be $u$-relatively uniformly convergent to $x \in X$ if for every $\varepsilon>0$ there exists $N \in \mathbb{N}$ such that for every $n \geq N$ we have $\pm\left(x_{n}-x\right) \leq \varepsilon u$. Then $x$ is called the $u$-relatively uniform limit of $\left(x_{n}\right)_{n}$. For $u \in K$, the sequence $\left(x_{n}\right)_{n}$ is called a $u$-relatively uniformly Cauchy sequence if for every $\varepsilon>0$ there exists $N \in \mathbb{N}$ such that $\pm\left(x_{m}-x_{n}\right) \leq \varepsilon u$ for every $m, n \geq N$. An ordered vector space $X$ is called uniformly complete if, for every $u \in K$, every $u$-relatively uniformly Cauchy sequence has a $u$-relatively uniform limit. Note that a uniformly complete vector lattice is Archimedean.

An element $u>0$ is called an order unit if for every $x \in X$ there is $\lambda \in \mathbb{R}, \lambda>0$, such that $-\lambda u \leq x \leq \lambda u$. If an ordered vector space has an order unit, then the space is directed. If $X$ is an Archimedean ordered vector space with order unit $u$, then

$$
\|\cdot\|_{u}: X \rightarrow[0, \infty), \quad x \mapsto\|x\|_{u}:=\inf \{\lambda \in(0, \infty) ;-\lambda u \leq x \leq \lambda u\}
$$

defines a norm on $X$, the so-called $u$-norm. In this case, the uniform completion of $X$ is defined to be the norm completion of $X$ with respect to the $u$-norm. The space $X$ is uniformly complete if and only if $X$ is norm complete with respect to the $u$-norm. 


\section{Examples}

This section shall illustrate that the theory of disjointness preserving operators in preRiesz spaces has aspects similar to the vector lattice setting, and aspects that are very different.

Concerning disjointness preserving operators in vector lattices, Riesz homomorphisms are typical examples. In pre-Riesz spaces, the following similar notion is introduced in [15].

Definition 3.1 Let $X$ and $Y$ be pre-Riesz spaces. An operator $T: X \rightarrow Y$ is said to be a Riesz* homomorphism if for every $x_{1}, \ldots, x_{n} \in X$ one has $T\left(\left\{x_{1}, \ldots, x_{n}\right\}^{\mathrm{ul}}\right) \subseteq$ $\left\{T x_{n}, \ldots, T x_{n}\right\}^{\mathrm{ul}}$.

In Riesz spaces, Riesz* homomorphisms and Riesz homomorphisms coincide; see [10, Lemma 2.3.2]. In pre-Riesz theory, Riesz* homomorphisms play a crucial role since they are exactly those operators that can be extended to Riesz homomorphisms between the corresponding Riesz completions; see the next statement.

Theorem 3.2 [15, Theorem 5.6] Let $X$ and $Y$ be pre-Riesz spaces and $\left(X^{\rho}, i_{X}\right)$ and $\left(Y^{\rho}, i_{Y}\right)$ their Riesz completions, respectively. Let $T: X \rightarrow Y$ be a linear map. Then the following statements are equivalent:

(i) $T$ is a Riesz * homomorphism.

(ii) There exists a Riesz homomorphism $T_{\rho}: X^{\rho} \rightarrow Y^{\rho}$ that extends $T$ in the sense that

$$
T_{\rho} \circ i_{X}=i_{Y} \circ T
$$

One expects that in pre-Riesz spaces Riesz* homomorphisms preserve disjointness.

Theorem 3.3 Let $X$ and $Y$ be pre-Riesz spaces and $T: X \rightarrow Y$ a Riesz* homomorphism. Then $T$ is a (positive) disjointness preserving operator.

Proof If $\left(X^{\rho}, i_{X}\right)$ and $\left(Y^{\rho}, i_{Y}\right)$ are the Riesz completions of $X$ and $Y$, respectively, then due to Theorem 3.2 there is a Riesz homomorphism $T_{\rho}: X^{\rho} \rightarrow Y^{\rho}$ that satisfies (1). In particular, $T_{\rho}$ is positive and disjointness preserving. Since $i_{X}$ and $i_{Y}$ are bipositive, $T$ is positive. Moreover, due to Theorem 2.3 one obtains that $T$ is disjointness preserving.

It is a remarkable fact in the theory of vector lattices, that every order bounded and disjointness preserving operator is regular [5]. However, this is not true for operators on pre-Riesz spaces, in general. In [14] an example of an order bounded, nonregular linear functional on a directed partially ordered vector space was given. We will use this example to construct an order bounded disjointness preserving operator which is not regular.

Example 3.4 For $A \subseteq[0, \infty)$ let $\chi_{A}$ denote the corresponding indicator function. Define for $n, k \in \mathbb{N}$, 


$$
\begin{aligned}
e_{n}:[0, \infty) & \rightarrow \mathbb{R}, t \mapsto \chi_{[n-1, n)}(t), \\
u_{n, k}:[0, \infty) & \rightarrow \mathbb{R}, \quad t \mapsto n t \chi_{\left[0, \frac{1}{n}\right]}(t)+\frac{1}{k} \chi_{\left\{n+\frac{1}{k}\right\}}(t),
\end{aligned}
$$

and consider the subspace $X:=\operatorname{span}\left\{e_{n}, u_{n, k} ; n, k \in \mathbb{N}\right\}$ of $\mathbb{R}^{[0, \infty)}$ with pointwise order. For every $x \in X$ there exists $t_{0}>0$ such that $x$ is affine and, hence, differentiable on $\left(0, t_{0}\right)$. Define

$$
T: X \rightarrow X, \quad x \mapsto\left(\lim _{t \downarrow 0} x^{\prime}(t)\right) e_{1}
$$

For sake of completeness, we list all relevant properties of $X$ and $T$, where (i), (iv) and (v) are already dealt with in [14].

(i) $X$ is directed. Indeed, every element in $X$ is bounded and has bounded support. For $x, y \in X$ there is $n \in \mathbb{N}$ such that $x, y \leq n \sum_{i=1}^{n} e_{i}$, hence $X$ is directed.

(ii) $X$ is a pre-Riesz space. Indeed, $\mathbb{R}^{[0, \infty)}$ is Archimedean, therefore its subspace $X$ is Archimedean as well. [15, Theorem 1.7(ii)] yields that $X$ is a pre-Riesz space.

(iii) $T$ is disjointness preserving. Indeed, let $x^{(1)}, x^{(2)} \in X$ with $x^{(1)} \perp x^{(2)}$. There is $M \in \mathbb{N}$ such that for $i \in\{1,2\}$

$$
x^{(i)}=\sum_{n=1}^{M} \alpha_{n}^{(i)} e_{n}+\sum_{n, k=1}^{M} \lambda_{n, k}^{(i)} u_{n, k},
$$

i.e. $x^{(1)}$ and $x^{(2)}$ are affine on $\left[0, \frac{1}{M}\right]$. Let without loss of generality $x^{(1)}=0$ on $\left[0, \frac{1}{M}\right]$, then $T x^{(1)}=0$ and hence $T x^{(1)} \perp T x^{(2)}$.

(iv) $T$ is order bounded. Indeed, for $v, w \in X$ with $v \leq w$ there are $N \in \mathbb{N}$ and $C \in(0, \infty)$ such that $\pm v, \pm w \leq C \sum_{i=1}^{N} e_{i}$.

An element $x \in X$ with $v \leq x \leq w$ is affine on $\left[0, \frac{1}{N}\right]$, hence $-2 N C e_{1} \leq T x \leq$ $2 N C e_{1}$.

(v) $T$ is not regular. Indeed, assume that there is a positive linear operator $S: X \rightarrow X$ such that $S \geq T$. For $n, k \in \mathbb{N}$ one has that $0 \leq u_{n, k} \leq e_{1}+\frac{1}{k} e_{n+1}$, hence

$$
T\left(u_{n, k}\right)=n e_{1} \leq S\left(u_{n, k}\right) \leq S\left(e_{1}\right)+\frac{1}{k} S\left(e_{n+1}\right),
$$

and therefore $k\left(n e_{1}-S\left(e_{1}\right)\right) \leq S\left(e_{n+1}\right)$ for every $k \in \mathbb{N}$. Since $X$ is Archimedean, it follows that $n e_{1}-S\left(e_{1}\right) \leq 0$. From $n e_{1} \leq S\left(e_{1}\right)$ for every $n \in \mathbb{N}$ one obtains $e_{1} \leq 0$, a contradiction.

\section{Disjointness preserving operators for a particular polyhedral cone}

In [10, Example 4.4.18], a four-ray cone in $\mathbb{R}^{3}$ is investigated and all disjoint elements are calculated. We generalize this example to $\mathbb{R}^{n}$ with a $(2 n-2)$-ray cone. It turns out that the dual cone has a base which is an $(n-1)$-dimensional hypercube. 
Example 4.1 Let $X=\mathbb{R}^{n}$ with $n \geq 3$ and let $e^{(i)}$ denote the standard basis vectors. For $i \in\{1, \ldots, n-1\}$, define $x^{(i)}:=e^{(i)}+e^{(n)}$ and $y^{(i)}:=-e^{(i)}+e^{(n)}$ and let

$$
K:=\operatorname{pos}\left\{x^{(1)}, \ldots, x^{(n-1)}, y^{(1)}, \ldots, y^{(n-1)}\right\} .
$$

Then $K$ is a cone in $X$. We endow $X$ with the partial order induced by $K$. It is clear that $K$ is closed, and that $e^{(n)}$ is an interior point of $K$ and hence an order unit of $(X, K)$. Consequently, $K$ is generating. As $K$ is closed and hence Archimedean, $(X, K)$ is a pre-Riesz space. We will determine the disjoint elements in $X$. We begin by showing that the dual cone

$$
K^{\prime}=\{f: X \rightarrow \mathbb{R}: f \text { is linear and } f(K) \subseteq[0, \infty)\}
$$

satisfies

$$
K^{\prime}=\operatorname{pos}\left\{f_{\sigma}: \sigma \in\{-1,1\}^{n-1}\right\}
$$

where

$$
f_{\sigma}(x):=\sum_{j=1}^{n-1} \sigma_{j} x_{j}+x_{n}, \quad x=\left(x_{1}, \ldots, x_{n}\right) \in X .
$$

Indeed, it is clear that for every $\sigma=\left(\sigma_{1}, \ldots, \sigma_{n-1}\right) \in\{-1,1\}^{n-1}$ and $i \in\{1, \ldots, n-$ 1 ) we have $f_{\sigma}\left(x^{(i)}\right) \geq 0$ and $f_{\sigma}\left(y^{(i)}\right) \geq 0$, so $f_{\sigma}$ is positive. Hence

$$
L:=\operatorname{pos}\left\{f_{\sigma}: \sigma \in\{-1,1\}^{n-1}\right\} \subseteq K^{\prime}
$$

Next, let $x \in X$ be such that $f_{\sigma}(x) \geq 0$ for every $\sigma \in\{-1,1\}^{n-1}$. We show that $x \in K$. For every $\sigma \in\{-1,1\}^{n-1}$, from

$$
0 \leq f_{\sigma}(x)=\sum_{i=1}^{n-1} \sigma_{i} x_{i}+x_{n}
$$

it follows that

$$
x_{n} \geq \sum_{i=1}^{n-1}-\sigma_{i} x_{i}
$$

and hence

$$
x_{n} \geq \sum_{i=1}^{n-1}\left|x_{i}\right|
$$


As $2 e^{(i)}=x^{(i)}-y^{(i)}$ and $2 e^{(n)}=x^{(i)}+y^{(i)}$ for every $i \in\{1, \ldots, n-1\}$, it follows that

$$
\begin{aligned}
2 x & =2 \sum_{i=1}^{n} x_{i} e^{(i)}=\sum_{i=1}^{n-1} x_{i}\left(x^{(i)}-y^{(i)}\right)+2 x_{n} e^{(n)} \\
& =\sum_{i=1}^{n-1}\left(\left(x_{i}+\left|x_{i}\right|\right) x^{(i)}+\left(\left|x_{i}\right|-x_{i}\right) y^{(i)}\right)-\sum_{i=1}^{n-1}\left|x_{i}\right|\left(x^{(i)}+y^{(i)}\right)+2 x_{n} e^{(n)} \\
& =\sum_{i=1}^{n-1}\left(x_{i}+\left|x_{i}\right|\right) x^{(i)}+\sum_{i=1}^{n-1}\left(\left|x_{i}\right|-x_{i}\right) y^{(i)}+2\left(x_{n}-\sum_{i=1}^{n-1}\left|x_{i}\right|\right) e^{(n)} \in K .
\end{aligned}
$$

Hence $x \in K$.

Now suppose that there exists $f \in K^{\prime} \backslash L$. Then, according to the Hahn-Banach separation theorem, there exist $x \in X$ and $c \in \mathbb{R}$ such that $g(x) \geq c$ for every $g \in L$ and $f(x)<c$. Then $c \leq 0$. For $g \in L$ and $n \in \mathbb{N}$ we have $n g \in L$, so $n g(x) \geq c$, so $g(x) \geq \frac{c}{n}$, hence $g(x) \geq 0$. In particular, $f_{\sigma}(x) \geq 0$ for every $\sigma \in\{-1,1\}^{n-1}$, so, by the previous step, we have $x \in K$. This contradicts $f(x)<c \leq 0$ and $f \in K^{\prime}$. Thus, $K^{\prime}=L$. Consequently, $K$ has the representation

$$
K=\left\{x \in \mathbb{R}^{n}: f_{\sigma}(x) \geq 0, \sigma \in\{-1,1\}^{n-1}\right\} .
$$

Next we determine the Riesz completion by means of the functional representation discussed in [10, Section 2.5]. Let

$$
\Sigma:=\left\{f \in K^{\prime}: f\left(e^{(n)}\right)=1\right\}
$$

and denote the set of all extreme points of $\Sigma$ by

$$
\Lambda:=\operatorname{ext}(\Sigma)
$$

We establish that $\Lambda=\left\{f_{\sigma}: \sigma \in\{-1,1\}^{n-1}\right\}$. It is clear that for every $\sigma \in$ $\{-1,1\}^{n-1}$ we have $f_{\sigma}\left(e^{(n)}\right)=1$, i.e. $f_{\sigma} \in \Sigma$. As $K^{\prime}=L$, we obtain $\Lambda \subseteq$ $\left\{f_{\sigma}: \sigma \in\{-1,1\}^{n-1}\right\}$. It remains to show that for every $\sigma \in\{-1,1\}^{n-1}$ we have $f_{\sigma} \in \Lambda$. Indeed, let $g, h \in \Sigma$ be such that $f_{\sigma}=\lambda g+(1-\lambda) h$ for some $\lambda \in(0,1)$. Then $g\left(e^{(n)}\right)=h\left(e^{(n)}\right)=1$. For every $i \in\{1, \ldots, n-1\}$, on one hand we have

$$
\begin{aligned}
f_{\sigma}\left(x^{(i)}\right) & =\lambda g\left(e^{(i)}+e^{(n)}\right)+(1-\lambda) h\left(e^{(i)}+e^{(n)}\right) \\
& =\lambda g\left(e^{(i)}\right)+(1-\lambda) h\left(e^{(i)}\right)+1 .
\end{aligned}
$$

On the other hand,

$$
f_{\sigma}\left(x^{(i)}\right)=\sigma_{i}+1 .
$$


We obtain

$$
\sigma_{i}=\lambda g\left(e^{(i)}\right)+(1-\lambda) h\left(e^{(i)}\right)
$$

It follows from $f_{\sigma}\left(e^{(i)}\right)=\sigma_{i}$ that

$$
f_{\sigma}\left(e^{(i)}\right)=\lambda g\left(e^{(i)}\right)+(1-\lambda) h\left(e^{(i)}\right)
$$

Since $-e^{(n)} \leq e^{(i)} \leq e^{(n)}$ (with respect to the order induced by the cone $K$ ) and $g, h \in K^{\prime}$, we get $g\left(-e^{(n)}\right) \leq g\left(e^{(i)}\right) \leq g\left(e^{(n)}\right)$ and $h\left(-e^{(n)}\right) \leq h\left(e^{(i)}\right) \leq$ $h\left(e^{(n)}\right)$. As $g\left(e^{(n)}\right)=1$ and $h\left(e^{(n)}\right)=1$, we have $-1 \leq g\left(e^{(i)}\right) \leq 1$ and $-1 \leq$ $h\left(e^{(i)}\right) \leq 1$. Hence, if $\sigma_{i}=-1$, then $g\left(e^{(i)}\right)=h\left(e^{(i)}\right)=-1$, and if $\sigma_{i}=1$, then $g\left(e^{(i)}\right)=h\left(e^{(i)}\right)=1$. We conclude $f_{\sigma}=g=h$. Geometrically, we obtain $\Sigma$ as an $(n-1)$-dimensional hypercube.

It is clear that $\bar{\Lambda}=\Lambda$. Due to the functional representation given in [10, Theorem 2.6.2], the Riesz completion $X^{\rho}$ of $(X, K)$ is given by $\mathrm{C}(\Lambda)$. Since $\Lambda$ contains $2^{n-1}$ elements, the space $X^{\rho}$ is $\left(2^{n-1}\right)$-dimensional. We will identify $X^{\rho}$ with $\mathbb{R}^{2^{n-1}}$, endowed with the standard cone. The corresponding bipositive linear map $\mathcal{I}: X \rightarrow X^{\rho}$ is given by

$$
\mathcal{I}: x \mapsto \sum_{j=1}^{2^{n-1}} f_{\gamma(j)}(x) e^{(j)}
$$

where $\gamma:\left\{1, \ldots, 2^{n-1}\right\} \rightarrow\left\{\sigma: \sigma \in\{-1,1\}^{n-1}\right\}$ is a bijection.

Next, we compute all directed and nondirected bands in the space of the previous example.

Example 4.2 We consider the pre-Riesz space $(X, K)$ as in Example 4.1 and proceed by determining all nontrivial disjoint elements. Denote for $i, j \in\{1, \ldots, n\}$ with $i<j$,

$$
\begin{aligned}
& x^{(i j)}:=e^{(i)}+e^{(j)}, \quad y^{(i j)}:=e^{(i)}-e^{(j)}, \\
& X^{(i j)}:=\operatorname{span}\left\{x^{(i j)}\right\}, \quad Y^{(i j)}:=\operatorname{span}\left\{y^{(i j)}\right\} .
\end{aligned}
$$

With the notation as in Example 4.1, observe that $x^{(i n)}=x^{(i)}$ and $y^{(i n)}=-y^{(i)}$ for $i \in\{1, \ldots, n-1\}$.

We will show that the nontrivial bands in $(X, K)$ are exactly the subspaces $X^{(i j)}$ and $Y^{(i j)}$ with $i, j \in\{1, \ldots, n\}, i<j$, where $\left(X^{(i j)}\right)^{\mathrm{d}}=Y^{(i j)}$. The proof consists of the following steps (a)-(g). To simplify the proof, we use that for $x \in X$ we have

$$
(\forall f \in \Lambda: f(x)=0) \Longleftrightarrow(\forall g \in(\Lambda \cup-\Lambda): g(x)=0)
$$


In view of this, we define for $\Theta \in\{-1,1\}^{n}$ the functional

$$
g_{\Theta}: X \rightarrow \mathbb{R}, \quad x \mapsto \sum_{i=1}^{n} \Theta_{i} x_{i}
$$

By Theorem 2.3, for $x, y \in X$ we have

$$
x \perp y \Longleftrightarrow \mathcal{I}(x) \perp \mathcal{I}(y)
$$

hence

$$
x \perp y \Longleftrightarrow \forall \Theta \in\{-1,1\}^{n}:\left(g_{\Theta}(x)=0 \text { or } g_{\Theta}(y)=0\right) \text {. }
$$

For $\Theta \in\{-1,1\}^{n}$ and $I \subseteq\{1, \ldots, n\}$ define $\Theta^{I} \in\{-1,1\}^{n}$ by

$$
\Theta_{i}^{I}:=-\Theta_{i} \text { for } i \in I, \quad \text { and } \Theta_{i}^{I}:=\Theta_{i} \text { otherwise. }
$$

(a) For $i, j \in\{1, \ldots, n\}$ with $i<j$ we have $x^{(i j)} \perp y^{(i j)}$. Indeed, for every $\Theta \in$ $\{-1,1\}^{n}$ we get $g_{\Theta}\left(x^{(i j)}\right)=\Theta_{i}+\Theta_{j}$ and $g_{\Theta}\left(y^{(i j)}\right)=\Theta_{i}-\Theta_{j}$. We have $\Theta_{i}+\Theta_{j} \neq 0$ if and only if $\Theta_{i}-\Theta_{j}=0$. Hence $g_{\Theta}\left(x^{(i j)}\right)=0$ or $g_{\Theta}\left(y^{(i j)}\right)=0$, so that $x^{(i j)} \perp y^{(i j)}$.

(b) The following technical observation will be needed several times later on. Let $x \in X$ and $\Theta \in\{-1,1\}^{n}$ be such that $g_{\Theta}(x)=0$. If $i \in\{1, \ldots, n\}$ is such that $g_{\Theta}\{i\}(x)=0$, then $x_{i}=0$. Indeed,

$$
\begin{aligned}
\left|2 x_{i}\right| & =\left|2 \Theta_{i} x_{i}\right|=\left|\left(\Theta_{i}-\Theta_{i}^{\{i\}}\right) x_{i}\right|=\left|\sum_{j=1}^{n}\left(\Theta_{j}-\Theta_{j}^{\{i\}}\right) x_{j}\right| \\
& =\left|g_{\Theta}(x)-g_{\Theta}\{i\}(x)\right|=0 .
\end{aligned}
$$

(c) For every $i, j \in\{1, \ldots, n\}$ with $i<j$ we have $\left\{x^{(i j)}\right\}^{\mathrm{d}} \subseteq Y^{(i j)}$. For a proof, let $y \in\left\{x^{(i j)}\right\}^{\mathrm{d}}$. For every $\Theta \in\{-1,1\}^{n}$ with $\Theta_{i}=\Theta_{j}=1$ we have $g_{\Theta}\left(x^{(i j)}\right)=$ 2 , so $g_{\Theta}(y)=0$. We fix $\Theta:=(1, \ldots, 1)$ to be the constant 1 vector. Then $g_{\Theta}(y)=0$. Moreover, for every $k \in\{1, \ldots, n\} \backslash\{i, j\}$ we have $\Theta_{i}^{\{k\}}=\Theta_{j}^{\{k\}}=1$, so $g_{\Theta}\{k\}(y)=0$. By (b), $y_{k}=0$. Then $g_{\Theta}(y)=0$ yields that $y_{i}+y_{j}=0$, so that $y \in Y^{(i j)}$.

(d) Similar to (c), for every $i, j \in\{1, \ldots, n\}$ with $i<j$ we have $\left\{y^{(i j)}\right\}^{\mathrm{d}} \subseteq X^{(i j)}$. Hence $\left\{x^{(i j)}\right\}^{\mathrm{d}} \subseteq Y^{(i j)} \subseteq\left(X^{(i j)}\right)^{\mathrm{d}} \subseteq\left\{x^{(i j)}\right\}^{\mathrm{d}}$. We conclude that $X^{(i j)}$ and $Y^{(i j)}$ are bands and that $\left(X^{(i j)}\right)^{\mathrm{d}}=Y^{(i j)}$. In the following three steps we show that these are the only nontrivial bands in $(X, K)$.

(e) Let $x \in X$ be such that there are $i, j \in\{1, \ldots, n\}$ with $i<j$ and $x_{i} \neq 0, x_{j} \neq 0$ and $x_{k}=0$ for every $k \in\{1, \ldots, n\} \backslash\{i, j\}$ and such that $\{x\}^{\mathrm{d}}$ is nontrivial. Then $x \in X^{(i j)}$ or $x \in Y^{(i j)}$. Indeed, as $\{x\}^{\mathrm{d}}$ is nontrivial, there is $\Theta \in\{-1,1\}^{n}$ such 
that $g_{\Theta}(x)=0$. Hence $\Theta_{i} x_{i}+\Theta_{j} x_{j}=0$, which yields that $x_{i}-x_{j}=0$ or $x_{i}+x_{j}=0$, so that $x \in X^{(i j)}$ or $x \in Y^{(i j)}$.

(f) Let $x \in X$ be such that there is $i \in\{1, \ldots, n\}$ with $x_{i} \neq 0$ and $x_{k}=0$ for every $k \in\{1, \ldots, n\} \backslash\{i\}$. Then $\{x\}^{\mathrm{d}}=\{0\}$. Indeed, for every $\Theta \in\{-1,1\}^{n}$ we have $g_{\Theta}(x)=\Theta_{i} x_{i} \neq 0$. Hence, if $y \in\{x\}^{\mathrm{d}}$, then $g_{\Theta}(y)=0$. So, $y=0$.

(g) Let $x \in X$ be such that at least three coordinates of $x$ are nonzero. Then $\{x\}^{\mathrm{d}}=$ $\{0\}$. Indeed, let $I:=\left\{i \in\{1, \ldots, n\}: x_{i} \neq 0\right\}$. Then $I$ has at least three elements. Let $y \in\{x\}^{\mathrm{d}}$ and suppose that $y \neq 0$. Then there is $\Theta \in\{-1,1\}^{n}$ such that $g_{\Theta}(y) \neq 0$, so $g_{\Theta}(x)=0$. For every $i \in I$ we have $g_{\Theta}\{i\}(x) \neq 0$ as $x_{i} \neq 0$, so $g_{\Theta}\{i\}(y)=0$. We need an intermediate claim.

(*) If $i, j \in I$ with $i \neq j$ are such that $\operatorname{sign}\left(\Theta_{i} x_{i}\right)=\operatorname{sign}\left(\Theta_{j} x_{j}\right)$, then $y_{i}=y_{j}=0$.

For a proof of $(*)$, note that $\operatorname{sign}\left(\Theta_{i} x_{i}\right)=\operatorname{sign}\left(\Theta_{j} x_{j}\right)$ yields $g_{\Theta}\{i, j\}(x) \neq g_{\Theta}(x)=0$, so $g_{\Theta}\{i, j\}(y)=0$. By (b), we obtain $y_{i}=y_{j}=0$.

Now we use $(*)$ to prove that $y=0$, which is a contradiction. Let $i \in I$. We show $y_{i}=0$. If $i \in I$ is such that there exists $j \in I \backslash\{i\}$ with $\operatorname{sign}\left(\Theta_{i} x_{i}\right)=\operatorname{sign}\left(\Theta_{j} x_{j}\right)$, then (*) gives $y_{i}=0$. Otherwise, for every $j \in I \backslash\{i\}$ we have $\operatorname{sign}\left(\Theta_{i} x_{i}\right) \neq \operatorname{sign}\left(\Theta_{j} x_{j}\right)$ and then $\operatorname{sign}\left(\Theta_{j} x_{j}\right)=\operatorname{sign}\left(\Theta_{k} x_{k}\right)$ for every $j, k \in I \backslash\{i\}$. As $I \backslash\{i\}$ contains at least two elements, $(*)$ yields that $y_{j}=0$ for every $j \in I \backslash\{i\}$. Then $g_{\Theta}\{i\}(y)=g_{\Theta I}(y)$, so $g_{\Theta^{I}}(y)=0$. Also, for $j \in I \backslash\{i\}$ we have $g_{\Theta}\{j\}(y)=g_{\Theta^{I \backslash \backslash i\}}}(y)$, as $y_{k}=0$ for every $k \in I \backslash\{i\}$. Hence, $g_{\Theta} I(y)=0$ and $g_{\Theta} \backslash\{i\}(y)=0$, so by (b) we obtain $y_{i}=0$.

Now let $i \in\{1, \ldots, n\} \backslash I$. Fix $j \in I$. We have $x_{i}=0$, so $g_{\Theta}\{i, j\}(x)=g_{\Theta}\{j\}(x) \neq 0$, hence $g_{\Theta}\{i, j\}(y)=0$. As also $g_{\Theta}\{j\}(y)=0$, (b) yields that $y_{i}=0$. Thus, we arrive at $y=0$, a contradiction.

From the statements in (e), (f) and (g) we conclude that only for elements of the bands $X^{(i j)}$ and $Y^{(i j)}$ there exist nontrivial disjoint elements. Thus, in $(X, K)$ there are only one-dimensional nontrivial bands. By construction, we have $n(n-1)$ nontrivial bands in $(X, K)$. Note that $X^{(i j)}$ and $Y^{(i j)}$ are directed, respectively, if and only if $j=n$. Hence there are $2(n-1)$ directed nontrivial bands and $(n-1)(n-2)$ nondirected nontrivial bands in $(X, K)$.

We determine all disjointness preserving bijective operators in $(X, K)$.

Example 4.3 For the pre-Riesz space $(X, K)$ in Example 4.1, we show that a linear bijection $T: X \rightarrow X$ is disjointness preserving if and only if there is a permutation $P:\{1, \ldots, n\} \rightarrow\{1, \ldots, n\}, \Theta \in\{-1,1\}^{n}$ and $\alpha \in \mathbb{R} \backslash\{0\}$ such that for every $j \in\{1, \ldots, n\}$ the $j$-th column of $T$ equals $\alpha \Theta_{j} e^{(P(j))}$. First we show that every operator $T$ of this type is disjointness preserving, where we use the notation and results of Example 4.2. Indeed, for every $i, j \in\{1, \ldots, n\}$ with $i<j$ we have

$$
\begin{aligned}
& T\left(x^{(i j)}\right)=T\left(e^{(i)}+e^{(j)}\right)=\alpha\left(\Theta_{i} e^{(P(i))}+\Theta_{j} e^{(P(j))}\right), \\
& T\left(y^{(i j)}\right)=T\left(e^{(i)}-e^{(j)}\right)=\alpha\left(\Theta_{i} e^{(P(i))}-\Theta_{j} e^{(P(j))}\right),
\end{aligned}
$$

hence $T\left(x^{(i j)}\right) \perp T\left(y^{(i j)}\right)$. Thus, $T$ is disjointness preserving.

Conversely, let $T: X \rightarrow X$ be a disjointness preserving bijection. For $j \in$ $\{1, \ldots, n\}$ let $T^{(j)}$ denote the $j$-th column of $T$. Let $k, l \in\{1, \ldots, n\}$ with $k<l$. 
Since $x^{(k l)}$ and $y^{(k l)}$ are disjoint and nonzero, we have that $T\left(x^{(k l)}\right)$ and $T\left(y^{(k l)}\right)$ are disjoint and nonzero. Hence, there are $i, j \in\{1, \ldots, n\}$ with $i<j, \theta \in\{-1,1\}$ and $\lambda, \mu \in \mathbb{R}$ such that $T\left(x^{(k l)}\right)=\lambda\left(e^{(i)}+\theta e^{(j)}\right)$ and $T\left(y^{(k l)}\right)=\mu\left(e^{(i)}-\theta e^{(j)}\right)$. Therefore

$$
\begin{aligned}
& 2 T^{(k)}=T^{(k)}+T^{(l)}+T^{(k)}-T^{(l)}=(\lambda+\mu) e^{(i)}+\theta(\lambda-\mu) e^{(j)}, \text { and } \\
& 2 T^{(l)}=T^{(k)}+T^{(l)}-\left(T^{(k)}-T^{(l)}\right)=(\lambda-\mu) e^{(i)}+\theta(\lambda+\mu) e^{(j)}
\end{aligned}
$$

If possible, choose $m \in\{1, \ldots, n\}$ with $k<m$ and $m \neq l$. Then $x^{(k m)}$ and $y^{(k m)}$ are disjoint and nonzero. (If such an $m$ does not exist, we choose $m<k$ and consider $x^{(m k)}$ and $y^{(m k)}$ instead.) As before, there are $p, q \in\{1, \ldots, n\}$ with $p<q$ and $\{p, q\} \neq\{i, j\}, \delta \in\{-1,1\}$, and $\alpha, \beta \in \mathbb{R}$ such that $T\left(x^{(k m)}\right)=\alpha\left(e^{(p)}+\delta e^{(q)}\right)$ and $T\left(y^{(k m)}\right)=\beta\left(e^{(p)}-\delta e^{(q)}\right)$. Consequently,

$$
\begin{aligned}
& 2 T^{(k)}=T^{(k)}+T^{(m)}+T^{(k)}-T^{(m)}=(\alpha+\beta) e^{(p)}+\delta(\alpha-\beta) e^{(q)}, \text { and } \\
& 2 T^{(m)}=T^{(k)}+T^{(m)}-\left(T^{(k)}-T^{(m)}\right)=(\alpha-\beta) e^{(p)}+\delta(\alpha+\beta) e^{(q)} .
\end{aligned}
$$

Without loss of generality, we assume $q \notin\{i, j\}$. Then $T^{(k)}=\alpha e^{(p)}$. We distinguish three cases.

(i) If $p \notin\{i, j\}$, then $T^{(k)}=0$, which is not possible since $T$ is a bijection.

(ii) If $p=i$, then $\lambda=\mu$, so $T^{(k)}=\lambda e^{(i)}$, hence $\lambda=\alpha$. Then $T^{(l)}=\theta \lambda e^{(j)}=$ $\theta \alpha e^{(j)}$.

(iii) If $p=j$, then $\mu=-\lambda$, so $T^{(k)}=\theta \lambda e^{(j)}$, hence $\theta \lambda=\alpha$. Then $T^{(l)}=\lambda e^{(i)}=$ $\theta \alpha e^{(i)}$.

Thus every two distinct columns of $T$ are different unit vectors multiplied by plus or minus the same scalar.

Next we determine the positive disjointness preserving bijections in $(X, K)$. Let $T$ be a disjointness preserving bijection in $(X, K)$, i.e. for every $j \in\{1, \ldots, n\}$ the $j$-th column of $T$ equals $\alpha \Theta_{j} e^{(P(j))}$, as above. We claim that $T$ is positive if and only if $\alpha>0$ and the $n$-th column of $T$ equals $\alpha e^{(n)}$. Indeed, assume that $T$ is positive. Since $x^{(1)}$ and $y^{(1)}$ are positive disjoint elements, there exists $i \in\{1, \ldots, n-1\}$ such that $T\left[\left\{x^{(1)}, y^{(1)}\right\}\right]=\left\{\alpha x^{(i)}, \alpha y^{(i)}\right\}$. Then

$$
2 T\left(e^{(n)}\right)=T\left(x^{(1)}+y^{(1)}\right)=\alpha x^{(i)}+\alpha y^{(i)}=2 \alpha e^{(n)} .
$$

Since $e^{(n)}$ is positive, it follows that $\alpha>0$ and that the $n$-th column of $T$ equals $\alpha e^{(n)}$.

Conversely, assume that $\alpha>0$ and that the $n$-th column of $T$ equals $\alpha e^{(n)}$. Let $i \in\{1, \ldots, n-1\}$. As $T\left(e^{(n)}\right)=\alpha e^{(n)}$, we obtain

$$
\begin{gathered}
T\left(x^{(i)}\right)=\alpha \theta_{i} e^{(P(i))}+\alpha e^{(n)}, \\
T\left(y^{(i)}\right)=-\alpha \theta_{i} e^{(P(i))}+\alpha e^{(n)} .
\end{gathered}
$$


Hence, $T\left(x^{(i)}\right)$ and $T\left(y^{(i)}\right)$ are positive. Thus $T$ maps each extreme ray of $K$ into $K$. Therefore, $T$ is positive.

Up to positive scalar multiples, there are $2^{n}(n !)$ disjointness preserving bijections in $(X, K)$. Among them, there are $2^{n-1}((n-1) !)$ positive ones.

\section{Pervasive pre-Riesz spaces as ranges}

Our aim in this section is to replace the range space $Y$ in Theorem 1.1 by a preRiesz space. The idea is to consider the Riesz completion $\left(Y^{\rho}, i\right)$ of $Y$ and apply Theorem 1.1 to $i \circ T$. For that purpose we need a Riesz norm on $Y^{\rho}$. The next lemma presents conditions on $Y$ that provide such a norm.

Lemma 5.1 Let $Y$ be a pervasive pre-Riesz space, let $(Z, i)$ be a vector lattice cover of $Y$, and let $\|\cdot\|_{Y}$ be a monotone norm on $Y$. Define for $z \in Z$

$$
\|z\|_{Z}=\inf \left\{\|y\|_{Y}: y \in Y, i(y) \geq|z|\right\} .
$$

Then $\|\cdot\|_{Z}$ is a Riesz, norm on Z. Moreover, for every $y \in Y$ we have $\|i(y)\|_{Z} \geq \frac{1}{2}\|y\|_{Y}$ and for every $y \in Y$ with $y \geq 0$ we have $\|i(y)\|_{Z}=\|y\|_{Y}$.

Proof By [13, Propositions 5.1 and 6.2], $\|\cdot\|_{Z}$ is a Riesz seminorm on $Z$ and both stated relations with $\|\cdot\|_{Y}$ hold. It remains to show that $\|\cdot\|_{Z}$ is a norm.

First observe that for $v \in Y, v \geq 0$, we have for every $y \in Y$ with $i(y) \geq|i(v)|$ that $y \geq v \geq 0$, hence $\|y\|_{Y} \geq\|v\|_{Y}$, so that $\|i(v)\|_{Z} \geq\|v\|_{Y}$.

Let $z \in Z$ be such that $z \neq 0$. Since $Y$ is pervasive, there exists $y \in Y$ with $0<i(y) \leq|z|$. Then $\|z\|_{Z}=\||z|\|_{Z} \geq\|i(y)\|_{Z} \geq\|y\|_{Y}>0$. Hence $\|\cdot\|_{Z}$ is a Riesz norm.

As a Riesz space with a Riesz norm is Archimedean, Lemma 5.1 yields that every pervasive pre-Riesz space with a monotone norm is Archimedean.

We can now extend Theorem 1.1 to a setting with the range space being a pre-Riesz space.

Theorem 5.2 Let $X$ be a uniformly complete vector lattice, $Y$ a pervasive pre-Riesz space with a monotone norm, and $T: X \rightarrow Y$ an injective and disjointness preserving operator. Then for every $x_{1}, x_{2} \in X$ we have that T $x_{1} \perp T x_{2}$ implies $x_{1} \perp x_{2}$.

Proof Let $\left(Y^{\rho}, i\right)$ be the Riesz completion of $Y$. Since $T: X \rightarrow Y$ is injective, we have that $i \circ T: X \rightarrow Y^{\rho}$ is injective as well. As $T$ is disjointness preserving, by means of Theorem 2.3 we have that $i \circ T$ is disjointness preserving. With the aid of Lemma 5.1, the monotone norm of $Y$ yields a Riesz norm on $Y^{\rho}$.

Let $x_{1}, x_{2} \in X$ be such that $T x_{1} \perp T x_{2}$. Then $(i \circ T) x_{1} \perp(i \circ T) x_{2}$. We apply Theorem 1.1 and obtain that $x_{1} \perp x_{2}$.

Corollary 5.3 Let X be a uniformly complete vector lattice, $Y$ a pervasive pre-Riesz space with a monotone norm, and $T: X \rightarrow Y$ a bijective and disjointness preserving operator. Then $T^{-1}: Y \rightarrow X$ is disjointness preserving as well. 
Proof Let $y_{1}, y_{2} \in Y$ be such that $y_{1} \perp y_{2}$. Take $x_{1}, x_{2} \in X$ with $T x_{1}=y_{1}$ and $T x_{2}=y_{2}$. We have $T x_{1} \perp T x_{2}$, hence according to Theorem 5.2 we obtain $x_{1} \perp x_{2}$. Therefore, $T^{-1}$ is disjointness preserving.

A key role in the proof of Theorem 1.1 is played by the next result due to de Pagter, see [4, Theorem 8].

Theorem 5.4 Let X be a uniformly complete Archimedean Riesz space and let $Y$ be an Archimedean Riesz space such that for every disjoint sequence $\left(w_{n}\right)_{n}$ in $Y$ with $w_{n}>0(n \in \mathbb{N})$ there exist positive real numbers $\lambda_{n}(n \in \mathbb{N})$ such that the set $\left\{\lambda_{n} w_{n}: n \in \mathbb{N}\right\}$ is not order bounded in $Y$. Then for every disjointness preserving operator $T: X \rightarrow Y$, there exists an order dense ideal in $X$ on which $T$ is order bounded.

One could try to generalize the range space in Theorem 5.4 to a more general preRiesz space $Y$ and thus try to generalize Theorem 1.1. It turns out that the conditions on $Y$ needed for this approach are not more general than those of the approach above. Nevertheless, our extension of Theorem 5.4 might be of independent interest.

We need the following simple observation, which follows from the fact that $i(Y)$ is majorizing in its Riesz completion $Y^{\rho}$.

Lemma 5.5 Let $Y$ be a pre-Riesz space and let $\left(Y^{\rho}, i\right)$ be its Riesz completion. For every subset $A \subseteq Y$, one has that $A$ is order bounded in $Y$ if and only if $i(A)$ is order bounded in $Y^{\rho}$.

We arrive at the following extension of Theorem 5.4.

Theorem 5.6 Let X be a uniformly complete Archimedean Riesz space and let $Y$ be a pervasive Archimedean pre-Riesz space such that for every disjoint sequence $\left(w_{n}\right)_{n}$ in $Y$ with $w_{n}>0(n \in \mathbb{N})$ there exist positive real numbers $\lambda_{n}(n \in \mathbb{N})$ such that the set $\left\{\lambda_{n} w_{n}: n \in \mathbb{N}\right\}$ is not order bounded in $Y$. Then for every disjointness preserving operator $T: X \rightarrow Y$, there exists an order dense ideal in $X$ on which $T$ is order bounded.

Proof Let $T: X \rightarrow Y$ be a disjointness preserving operator. Let $\left(Y^{\rho}, i\right)$ denote the Riesz completion of $Y$. By Theorem 2.3, we have for every $x_{1}, x_{2} \in X$ that $i\left(T x_{1}\right) \perp$ $i\left(T x_{2}\right)$ in $Y^{\rho}$ if and only if $T x_{1} \perp T x_{2}$ in $Y$, so $i \circ T: X \rightarrow Y^{\rho}$ is disjointness preserving as well.

Let $\left(w_{n}\right)_{n}$ be a disjoint sequence in $Y^{\rho}$ with $w_{n}>0(n \in \mathbb{N})$. Since $Y$ is pervasive, for every $n \in \mathbb{N}$ there exists $y_{n} \in Y$ with $0<i\left(y_{n}\right) \leq w_{n}$. Then $\left(y_{n}\right)_{n}$ is a disjoint sequence in $Y$, so there exist positive real numbers $\lambda_{n}(n \in \mathbb{N})$ such that $\left\{\lambda_{n} y_{n}: n \in \mathbb{N}\right\}$ is not order bounded in $Y$. With the aid of Lemma 5.5, it follows that $\left\{\lambda_{n} i\left(y_{n}\right): n \in \mathbb{N}\right\}$ is not order bounded and hence $\left\{\lambda_{n} w_{n}: n \in \mathbb{N}\right\}$ is not order bounded.

Theorem 5.4 now yields that there exists an order dense ideal $D$ in $X$ on which $i \circ T$ is order bounded. Then it follows from Lemma 5.5 that $T$ is order bounded on $D$.

The condition in Theorems 5.4 and 5.6 involving the disjoint sequence $\left(w_{n}\right)_{n}$ is satisfied if the space $Y$ can be equipped with a monotone norm. The next lemma provides the details of the simple verification of this fact. 
Lemma 5.7 If $Y$ is a pre-Riesz space with a monotone norm $\|\cdot\|_{Y}$, then for every sequence $\left(w_{n}\right)_{n}$ in $Y$ with $w_{n}>0(n \in \mathbb{N})$, there exist positive real numbers $\lambda_{n}$ $(n \in \mathbb{N})$ such that the set $\left\{\lambda_{n} w_{n}: n \in \mathbb{N}\right\}$ is not order bounded in $Y$.

Proof For the disjoint sequence $\left(w_{n}\right)_{n}$ in $Y$, let $\lambda_{n}:=\frac{n}{\left\|w_{n}\right\|_{Y}}$. Then $\left\|\lambda_{n} w_{n}\right\|_{Y}=$ $\frac{n}{\left\|w_{n}\right\|_{Y}}\left\|w_{n}\right\|_{Y}=n$, so $\left\{\lambda_{n} w_{n}: n \in \mathbb{N}\right\}$ is not norm bounded. Since the norm of $Y$ is monotone, every order bounded set in $Y$ is norm bounded. Hence $\left\{\lambda_{n} w_{n}: n \in \mathbb{N}\right\}$ is not order bounded in $Y$.

Observe that if $X$ is a Banach lattice then $X$ is a uniformly complete Archimedean Riesz space [11, Proposition 1.1.8(iv)]. By combining Theorem 5.6 with Lemma 5.7, we immediately obtain the following coro.

Corollary 5.8 If $X$ is a Banach lattice and $Y$ a pervasive pre-Riesz space with a monotone norm, then for every disjointness preserving operator $T: X \rightarrow Y$ there exists an order dense ideal in $X$ on which $T$ is order bounded.

\section{Extension of disjointness preserving operators on pre-Riesz spaces}

In this section, we investigate whether in Theorem 1.1 the uniformly complete vector lattice $X$ can be replaced by a more general pre-Riesz space. We try to accomplish such a generalization by extending the operator. Given a disjointness preserving operator on a pre-Riesz space, we wish to extend it to a disjointness preserving operator on its Riesz completion. This turns out to be a difficult task and our attempts only succeed under quite strong additional conditions. We start by identifying some denseness properties yielding that an operator that extends a disjointness preserving operator is itself disjointness preserving.

Theorem 6.1 Let $(X, K)$ be a pre-Riesz space with Riesz completion $\left(X^{\rho}, i\right)$. Let $Y$ be a vector lattice. Assume that for every $x \in X^{\rho}$ with $x \geq 0$, there exists a sequence $\left(x_{n}\right)_{n}$ in $K$ such that $i\left(x_{n}\right) \uparrow x$. If $T: X^{\rho} \rightarrow Y$ is an order continuous operator such that $(T \circ i): X \rightarrow Y$ is a positive disjointness preserving operator, then $T$ is also disjointness preserving.

Proof Let $x, y \in\left(X^{\rho}\right)^{+}$such that $x \perp y$. It suffices to show that $T x \perp T y$. By assumption, there exist sequences $\left(x_{n}\right)_{n}$ and $\left(y_{n}\right)_{n}$ in $K$ such that $i\left(x_{n}\right) \uparrow x$ and $i\left(y_{n}\right) \uparrow y$. Then clearly $i\left(x_{n}\right) \perp i\left(y_{n}\right)$, so $x_{n} \perp y_{n}$ in $X$ for every $n$. Since $T$ is order continuous and the lattice operations are order continuous [10, Proposition 1.1.34], we obtain that $T i\left(x_{n}\right) \wedge T i\left(y_{n}\right)$ converges to $T x \wedge T y$. Since

$$
T i\left(x_{n}\right) \wedge T i\left(y_{n}\right)=(T \circ i) x_{n} \wedge(T \circ i) y_{n}=0,
$$

it follows that $T x \perp T y$.

Theorem 6.2 Let $X$ be a pre-Riesz space with Riesz completion $\left(X^{\rho}, i\right)$ and let $\left(Y,\|\cdot\|_{Y}\right)$ be a normed vector lattice. Assume that there is a norm $\|\cdot\|_{X^{\rho}}$ on $X^{\rho}$ such that for every $x \in X^{\rho}$ with $x>0$, there exists a sequence $\left(x_{n}\right)_{n}$ in $X$ with 
$0 \leq i\left(x_{n}\right) \leq x$ for every $n$ and $\left\|i\left(x_{n}\right)-x\right\|_{X^{\rho}} \rightarrow 0$. If $T: X^{\rho} \rightarrow Y$ is norm continuous and $(T \circ i): X \rightarrow Y$ is a positive linear disjointness preserving operator, then $T$ is also disjointness preserving.

Proof Let $x, y$ in $\left(X^{\rho}\right)^{+}$with $x \perp y$. If $x=0$ or $y=0$, then it is clear that $T x \perp T y$. Assume that $x>0$ and $y>0$. By assumption, there exist sequences $\left(x_{n}\right)_{n}$ and $\left(y_{n}\right)_{n}$ in $X$ with $0 \leq i\left(x_{n}\right) \leq x$ and $0 \leq i\left(y_{n}\right) \leq y$ for every $n$, and $\| i\left(x_{n}\right)-$ $x\left\|_{X^{\rho}} \rightarrow 0,\right\| i\left(y_{n}\right)-y \|_{X^{\rho}} \rightarrow 0$. Then $x_{n} \perp y_{n}$ for all $n \in \mathbb{N}$, so that $(T \circ i)\left(x_{n}\right) \perp$ $(T \circ i)\left(y_{n}\right)$. Since $T$ is norm continuous, it follows that $\left\|(T \circ i)\left(x_{n}\right)-T(x)\right\|_{Y} \rightarrow 0$ and $\left\|(T \circ i)\left(y_{n}\right)-T(y)\right\|_{Y} \rightarrow 0$. As the lattice operations are norm continuous in the normed vector lattice $Y$ [10, Proposition 3.6.19], we have

$$
\left\|\left|(T \circ i)\left(x_{n}\right)\right| \wedge\left|(T \circ i)\left(y_{n}\right)\right|-|T(x)| \wedge|T(y)|\right\|_{Y} \rightarrow 0 .
$$

Therefore, $|T(x)| \wedge|T(y)|=0$, and hence $T$ is disjointness preserving.

Remark 6.3 In the Theorems 6.1 and 6.2, one can replace the Riesz completion $X^{\rho}$ by an arbitrary vector lattice cover. Since the Riesz completion is the smallest vector lattice cover, the condition on $X$ will be stronger for other vector lattice covers.

The next theorem presents a situation in which the existence of a norm in $X^{\rho}$ and continuity of $T$ as needed in Theorem 6.2 are satisfied.

Theorem 6.4 Let $X$ be a pervasive pre-Riesz space with Riesz completion $\left(X^{\rho}, i_{X}\right)$, let $\|\cdot\|_{X}$ be a monotone norm on $X$, and let $\|\cdot\|_{X^{\rho}}$ be defined as in (2). Let $Y$ be a partially ordered vector space with a monotone norm $\|\cdot\|_{Y}$, and let $T: X \rightarrow Y$ be a positive and continuous linear map. Then every positive linear map $\widehat{T}: X^{\rho} \rightarrow Y$ that extends $T$ in the sense that $\widehat{T} \circ i_{X}=T$ is continuous with respect to $\|\cdot\|_{X^{\rho}}$ and $\|\widehat{T}\| \leq\|T\|$.

Proof As $\|\cdot\|_{X}$ is monotone, it follows from Lemma 5.1 that $\|\cdot\|_{X^{\rho}}$ is a Riesz norm. Let $u \in X^{\rho}$ with $u \geq 0$. For $x \in X$ with $u \leq i(x)$ we have $0 \leq \widehat{T} u \leq T x$, as $T$ and $\widehat{T}$ are positive. So $\|\widehat{T} u\|_{Y} \leq\|T x\|_{Y} \leq\|T\|\|x\|_{X}$, hence $\|\widehat{T} u\|_{Y} \leq\|T\|\|u\|_{X^{\rho}}$. Thus, $\widehat{T}$ is continuous and $\|\widehat{T}\| \leq\|T\|$.

To extend a positive linear operator from a pre-Riesz space into a Dedekind complete vector lattice, we need the following extension theorem due to Kantorovich.

Theorem 6.5 (Kantorovich [11], Corollary 1.5.9) Let $X$ be an Archimedean Riesz space, $Y$ a Dedekind complete Riesz space. Every positive linear operator $T: D \rightarrow Y$ defined on a majorizing subspace $D$ of $X$ extends to all of $X$ as a positive linear operator.

Since a pre-Riesz space is a majorizing subspace of the Riesz completion, we could use Kantorovich's theorem to extend a positive operator from an Archimedean preRiesz space $X$ to a positive operator on the Riesz completion of $X$, provided the range space is Dedekind complete. 
Theorem 6.6 Let $X$ be a pervasive pre-Riesz space with Riesz completion $\left(X^{\rho}, i_{X}\right)$, let $\|\cdot\|_{X}$ be a monotone norm on $X$, and let $\|\cdot\|_{X^{\rho}}$ be the norm on $X^{\rho}$ defined as in (2). Assume that for every $x \in X^{\rho}$ with $x>0$ there exists a sequence $\left(x_{n}\right)_{n}$ in $X$ with $0 \leq i\left(x_{n}\right) \leq x$ for every $n$ and $\left\|i\left(x_{n}\right)-x\right\|_{X^{\rho}} \rightarrow 0$. Let $Y$ be a pervasive pre-Riesz space with Dedekind completion $\left(Y^{\delta}, i_{Y}\right)$, let $\|\cdot\|_{Y}$ be a monotone norm on $Y$, and let $\|\cdot\|_{Y^{\delta}}$ be the norm on $Y^{\delta}$ defined as in (2). If $T: X \rightarrow Y$ is a positive linear map that is continuous, disjointness preserving and injective, then there exists a positive linear extension $\widehat{T}: X^{\rho} \rightarrow Y^{\delta}$ of $i_{Y} \circ T \circ i_{X}^{-1}: i_{X}(X) \rightarrow Y^{\delta}$ that is continuous, disjointness preserving and injective as well. Moreover, if $X$ has an order unit and $X^{\rho u}$ denotes the uniform completion of $X^{\rho}$, then there exists a positive linear extension $T_{u}: X^{\rho u} \rightarrow Y^{\delta}$ of $i_{Y} \circ T \circ i_{X}^{-1}: i_{X}(X) \rightarrow Y^{\delta}$ that is disjointness preserving and injective.

Proof Due to Theorem 6.5, there exists a positive operator $\widehat{T}: X^{\rho} \rightarrow Y^{\delta}$ extending $i_{Y} \circ T \circ i_{X}^{-1}: i_{X}(X) \rightarrow Y^{\delta}$. By Theorem $6.4, \widehat{T}$ is continuous with respect to the Riesz norm $\|\cdot\|_{X^{\rho}}$ on $X^{\rho}$ and $\|\cdot\|_{Y^{\delta}}$ on $Y^{\delta}$. By Theorem 6.2, $\widehat{T}$ is disjointness preserving.

Next we show that $\widehat{T}$ is injective. Let $v \in X^{\rho}$ with $\widehat{T} v=0$; then $\widehat{T} v^{+}-\widehat{T} v^{-}=0$. As $\widehat{T}$ is disjointness preserving, we have $\widehat{T} v^{+}=0$ and $\widehat{T} v^{-}=0$. Suppose that $v \neq 0$; then either $v^{+} \neq 0$ or $v^{-} \neq 0$. Assume without loss of generality that $v^{+} \neq 0$. As $X$ is pervasive, there is an element $x \in X \backslash\{0\}$ with $0 \leq i_{X}(x) \leq v^{+}$. So we have $0 \leq i_{X}(T x)=\widehat{T} i_{X}(x) \leq \widehat{T} v^{+}=0$ and hence $T x=0$. This contradicts that $T$ is injective. Therefore $v=0$ and hence $\widehat{T}$ is injective. So $\widehat{T}: X^{\rho} \rightarrow Y^{\delta}$ is a positive disjointness preserving injective operator.

As $X^{\rho}$ is a majorizing subspace of $X^{\rho u}$, by Theorem $6.5, \widehat{T}$ can be extended to a positive operator $T_{u}: X^{\rho u} \rightarrow Y^{\delta}$.

We show as an intermediate step that every positive element in $X^{\rho u}$ can be approximated from below in the relative uniform topology by a sequence from $i(X)$. Let $u$ be an order unit in $X$. Then $i(u)$ is an order unit in $X^{\rho}$. For $z \in\left(X^{\rho u}\right)^{+}, z \neq 0$, there exist a sequence $\left(z_{n}\right)_{n} \in\left(X^{\rho}\right)^{+}$and a sequence $\left(\lambda_{n}\right)_{n}$ in $\mathbb{R}$ with $\lambda_{n} \downarrow 0$ and $\left|z_{n}-z\right| \leq \lambda_{n} i(u)$ for every $n \in \mathbb{N}$. Then $z_{n}-\lambda_{n} i(u) \leq z$ for every $n$. Take $w_{n}=\left(z_{n}-\lambda_{n} i(u)\right)^{+}$in $X^{\rho}$. We have $w_{n} \in X^{\rho}, 0 \leq w_{n} \leq z$, and

$$
\left|w_{n}-z\right|=\left|\left(z_{n}-\lambda i(u)\right)^{+}-z^{+}\right| \leq\left|z_{n}-\lambda_{n} i(u)-z\right| \leq 2 \lambda_{n} i(u),
$$

so $w_{n} \rightarrow z i(u)$-relatively uniformly.

Next we show that $T_{u}$ is disjointness preserving. Let $v, w \in X^{\rho u}$ with $|v| \perp|w|$. By the previous discussion, there exist sequences $\left(v_{n}\right)_{n}$ and $\left(w_{n}\right)_{n}$ in $X^{\rho}$ such that $0 \leq v_{n} \leq|x|, 0 \leq w_{n} \leq|y|$ and $v_{n} \rightarrow|v|$ and $w_{n} \rightarrow|w| i(u)$-relatively uniformly. Then $0 \leq v_{n} \wedge w_{n} \leq|v| \wedge|w|=0$, so $v_{n} \perp w_{n}$ and therefore $\widehat{T} v_{n} \perp \widehat{T} w_{n}$. Also, it follows that there exist sequences $\left(\alpha_{n}\right)$ and $\left(\beta_{n}\right)$ in $\mathbb{R}$ with $\alpha_{n} \downarrow 0$ and $\beta_{n} \downarrow 0$ such that $\left|v_{n}-\right| v|| \leq \alpha_{n} i(u)$ and $\left|w_{n}-\right| w|| \leq \beta_{n} i(u)$ for every $n$. We obtain that $|v| \leq|| v\left|-v_{n}\right|+\left|v_{n}\right| \leq \alpha_{n} i(u) u+v_{n}$ and $|w| \leq|| w\left|-w_{n}\right|+\left|w_{n}\right| \leq \beta_{n} i(u)+w_{n}$. It follows that

$$
\begin{aligned}
T_{u}(|v|) \wedge T_{u}(|w|) & \leq T_{u}\left(\alpha_{n} i(u)+v_{n}\right) \wedge T_{u}\left(\beta_{n} i(u)+w_{n}\right) \\
& \leq\left(\alpha_{n} T_{u}(i(u))+T_{u}\left(v_{n}\right)\right) \wedge\left(\beta_{n} T_{u}(i(u))+T_{u}\left(w_{n}\right)\right) \\
& \leq\left(\left(\alpha_{n} \vee \beta_{n}\right) T_{u}(i(u))+T_{u}\left(v_{n}\right)\right) \wedge\left(\left(\alpha_{n} \vee \beta_{n}\right) T_{u}(i(u))+T_{u}\left(w_{n}\right)\right)
\end{aligned}
$$




$$
\begin{aligned}
& =\left(\alpha_{n} \vee \beta_{n}\right) T_{u}(i(u))+\left(T_{u}\left(v_{n}\right) \wedge T_{u}\left(w_{n}\right)\right) \\
& =\left(\alpha_{n} \vee \beta_{n}\right) \widehat{T}(i(u))+\left(\widehat{T}\left(v_{n}\right) \wedge \widehat{T}\left(w_{n}\right)\right) \\
& =\left(\alpha_{n} \vee \beta_{n}\right) \widehat{T}(i(u)) .
\end{aligned}
$$

Since $Y^{\delta}$ is Archimedean and $\alpha_{n} \vee \beta_{n} \downarrow 0$, we infer that $T_{u}(|v|) \wedge T_{u}(|w|)=0$. Hence $T_{u}: X^{\rho u} \rightarrow Y^{\delta}$ is disjointness preserving.

Proposition 6.7 In the setting of Theorem $6.6, T_{u}: X^{\rho u} \rightarrow T_{u}\left(X^{\rho u}\right)$ has a disjointness preserving inverse $T_{u}^{-1}: T_{u}\left(X^{\rho u}\right) \rightarrow X^{\rho u}$.

Proof Note that $T_{u}$ is a positive disjointness preserving operator, hence a Riesz homomorphism. Therefore, $T_{u}\left(X^{\rho u}\right)$ is a Riesz subspace of $Y^{\delta}$. The norm of $Y$ yields a Riesz norm on $Y^{\delta}$ by (2). Since $T_{u}$ is injective, Corollary 5.3 yields that $T_{u}^{-1}: T_{u}\left(X^{\rho u}\right) \rightarrow X^{\rho u}$ is disjointness preserving.

If we combine Theorem 1.1 and Theorem 6.6, we obtain the following.

Theorem 6.8 In the setting of Theorem 6.6, if $T: X \rightarrow Y$ is bijective, then $T^{-1}$ is disjointness preserving.

In the following example, we will give an application of Theorem 6.6.

Example 6.9 Let $m \in \mathbb{N}$ and let $C^{m}[0,1]$ be the subspace of $C[0,1]$ consisting of all $m$ times continuously differentiable functions on $[0,1]$. For every $f \in C[0,1]^{+}$there exists a sequence $\left(f_{n}\right)_{n}$ in $C^{m}[0,1]^{+}$with $0 \leq f_{n} \leq f$ and $\left\|f_{n}-f\right\|_{\infty} \rightarrow 0$ and also $f_{n} \uparrow f$. We will give a proof of this statement in six steps below. The main idea is to approximate $f$ for a given $\varepsilon>0$ up to $6 \varepsilon$ from below by a $g \in C^{m}[0,1]^{+}$, by choosing $g$ to be 0 where $f \leq 4 \varepsilon$, choosing $g$ between $f-4 \varepsilon$ and $f-3 \varepsilon$ where $f>6 \varepsilon$ and glue these pieces of $g$ smoothly together. Some technical precautions are needed to make sure that our construction involves only finitely many subintervals and that the smooth connecting parts of $g$ are between 0 and $f$.

(a) Firstly, note that for every $f \in C[0,1]$ and every $\varepsilon>0$ there exists $g \in C^{m}[0,1]$ such that $\|f-g\|_{\infty}<4 \varepsilon$ and $g \leq f-3 \varepsilon$. Indeed, according to Weierstrass's approximation theorem, there exists $g \in C^{m}[0,1]$ such that $\|(f-(7 / 2) \varepsilon)-g\|_{\infty}<$ $\varepsilon / 2$ and then $g<(f-(7 / 2) \varepsilon)+\varepsilon / 2=f-3 \varepsilon$ and $\|f-g\|_{\infty}<4 \varepsilon$.

(b) Secondly, observe the following elementary gluing result. If $\varepsilon>0$ and $p, q, r, s \in[0,1]$ are such that $p<q<r<s$ and $g:[p, q] \cup[r, s] \rightarrow \mathbb{R}$ is a $C^{m}$ function, then there exists $h \in C^{m}[p, s]$ such that $h=g$ on $[p, q] \cup[r, s]$ and $\min \{g(q), g(r)\}-\varepsilon \leq h(t) \leq \max \{g(q), g(r)\}+\varepsilon$ for every $t \in(q, r)$.

(c) Thirdly, observe that the following variations on (b) are also true. If $\varepsilon>0$ and $p, q, r, s \in[0,1]$ are such that $p<q<r<s$ and $g:[p, q] \cup[r, s] \rightarrow \mathbb{R}$ is $C^{m}$, $g(q)>\varepsilon$, and $g=0$ on $[r, s]$, then there exists $h \in C^{m}[p, s]$ such that $h=g$ on $[p, q] \cup[r, s]$ and $0 \leq h(t)<g(q)+\varepsilon$ for every $t \in(q, r)$.

If $\varepsilon>0$ and $p, q, r, s \in[0,1]$ are such that $p<q<r<s$ and $g:[p, q] \cup[r, s] \rightarrow$ $\mathbb{R}$ is $C^{m}, g(r)>\varepsilon$, and $g=0$ on $[p, q]$ then there exists $h \in C^{m}[p, s]$ such that $h=g$ on $[p, q] \cup[r, s]$ and $0 \leq h(t)<g(r)+\varepsilon$ for every $t \in(q, r)$.

(d) Next, let $f \in C[0,1]^{+}$be such that $f(0)>0$ and $f(1)>0$ and let $\varepsilon>0$. We will construct a $g \in C^{m}[0,1]$ such that $0 \leq g \leq f$ and $\|g-f\|_{\infty} \leq 6 \varepsilon$. Without loss of generality we may assume that $\varepsilon$ is so small that $f(0)>6 \varepsilon$ and $f(1)>6 \varepsilon$. 
Define $\tau_{0}=0$ and for $k \in \mathbb{N}$ define, inductively,

$$
\begin{aligned}
\sigma_{k} & :=\inf \left\{t \in\left[\tau_{k-1}, 1\right]: f(t)<5 \varepsilon \text { or } t=1\right\}, \text { and } \\
\tau_{k} & :=\inf \left\{t \in\left[\sigma_{k}, 1\right]: f(t)>6 \varepsilon\right\} .
\end{aligned}
$$

We have $\sigma_{1}>0$ and for $k \in \mathbb{N}$ with $\sigma_{k}<1$ we have $\sigma_{k}<\tau_{k}$, since $f(0), f(1)>6 \varepsilon$ and $f$ is continuous. If $\sigma_{k}=1$, then $\tau_{k}=1$. Similarly, for every $k \in \mathbb{N}$ we have $\tau_{k}<\sigma_{k+1}$ or $\tau_{k}=\sigma_{k+1}=1$. For $k \in \mathbb{N}$ with $\sigma_{k}<1$ we have $f\left(\sigma_{k}\right)=5 \varepsilon$ and if $\tau_{k}<1$ then $f\left(\tau_{k}\right)=6 \varepsilon$. There exists $N \in \mathbb{N}$ such that $\sigma_{k}=\tau_{k}=1$ for every $k \geq N$, since otherwise there would be a convergent subsequence $\left(\sigma_{k_{j}}\right)_{j}$ with $\sigma_{k_{j}}<1$ and hence $f\left(\sigma_{k_{j}}\right)=5 \varepsilon$ for every $j$, and then $\left(\tau_{k_{j}}\right)_{j}$ would converge to the same limit while $f\left(\tau_{k_{j}}\right)=6 \varepsilon$, which contradicts the continuity of $f$.

Now we are ready to construct the desired function $g$. Let $k \in \mathbb{N}$ be such that $\tau_{k-1}<1$. On $\left[\tau_{k-1}, \sigma_{k}\right]$, we the aid of (a), we take $g$ to be $C^{m}$ and such that

$$
g \leq f-3 \varepsilon \text { on }\left[\tau_{k-1}, \sigma_{k}\right]
$$

and $\sup _{t \in\left[\tau_{k-1}, \sigma_{k}\right]}|f(t)-g(t)|<4 \varepsilon$. Since $f \geq 5 \varepsilon$ on $\left[\tau_{k-1}, \sigma_{k}\right]$, we have

$$
g>\varepsilon \text { on }\left[\tau_{k-1}, \sigma_{k}\right]
$$

If $\sigma_{1}=1$, then we have thus defined $g$ on all of $[0,1]$. Otherwise, let $k \in \mathbb{N}$ be such that $\sigma_{k}<1$. We will define $g$ on $\left(\sigma_{k}, \tau_{k}\right)$. Recall that $\tau_{k}<1$, so that $g$ has already been defined on $\left[\tau_{k-1}, \sigma_{k}\right] \cup\left[\tau_{k}, \sigma_{k+1}\right]$. Observe that $f \leq 6 \varepsilon$ on $\left[\sigma_{k}, \tau_{k}\right]$.

If $f \geq 4 \varepsilon$ on $\left[\sigma_{k}, \tau_{k}\right]$, then with the aid of step (b), we take $g$ on $\left(\sigma_{k}, \tau_{k}\right)$ such that $g$ is $C^{m}$ on $\left[\tau_{k-1}, \sigma_{k+1}\right]$ and $\min \left\{g\left(\sigma_{k}\right), g\left(\tau_{k}\right)\right\}-\varepsilon \leq g(t) \leq \max \left\{g\left(\sigma_{k}\right), g\left(\tau_{k}\right)\right\}+\varepsilon$ for every $t \in\left(\sigma_{k}, \tau_{k}\right)$. As $g\left(\sigma_{k}\right), g\left(\tau_{k}\right)>\varepsilon$, it follows that $g>0$ on $\left(\sigma_{k}, \tau_{k}\right)$. Since $g \leq f-3 \varepsilon \leq 3 \varepsilon$ at $\sigma_{k}$ and at $\tau_{k}$, we also have that $g(t)<4 \varepsilon \leq f$ for every $t \in\left(\sigma_{k}, \tau_{k}\right)$.

If we do not have that $f \geq 4 \varepsilon$ on $\left[\sigma_{k}, \tau_{k}\right]$, then we define

$$
\begin{aligned}
\pi_{k} & :=\inf \left\{t \in\left[\sigma_{k}, \tau_{k}\right]: f(t)<4 \epsilon\right\} \text { and } \\
\rho_{k} & :=\sup \left\{t \in\left[\pi_{k}, \tau_{k}\right]: f(t)<4 \varepsilon\right\} .
\end{aligned}
$$

Note that $\pi_{k}<\rho_{k}$ and $f \geq 4 \varepsilon$ on $\left[\sigma_{k}, \pi_{k}\right] \cup\left[\rho_{k}, \tau_{k}\right]$. On $\left[\pi_{k}, \rho_{k}\right]$ we take $g=0$. Recall that $g\left(\sigma_{k}\right)>\varepsilon$. With the aid of step (c), we take $g$ on $\left(\sigma_{k}, \pi_{k}\right)$ such that $g$ is a $C^{m}$ function on $\left[\tau_{k-1}, \rho_{k}\right]$ and $0 \leq g(t)<g\left(\sigma_{k}\right)+\varepsilon$ for every $t \in\left(\sigma_{k}, \pi_{k}\right)$. Then for every $t \in\left(\sigma_{k}, \pi_{k}\right)$ we have $g(t)<f\left(\sigma_{k}\right)-3 \varepsilon+\varepsilon=3 \varepsilon<f(t)$.

Similarly, on $\left(\rho_{k}, \tau_{k}\right)$ we take $g$ such that $g$ is $C^{m}$ on $\left[\pi_{k}, \sigma_{k+1}\right]$ and $0 \leq g(t)<$ $g\left(\tau_{k}\right)+\varepsilon$ for every $t \in\left(\rho_{k}, \tau_{k}\right)$. Then for every $t \in\left(\rho_{k}, \tau_{k}\right)$ we have $g(t)<f\left(\tau_{k}\right)-$ $3 \varepsilon+\varepsilon=4 \varepsilon \leq f(t)$. Thus, we have constructed $g$ on $\left(\sigma_{k}, \tau_{k}\right)$ such that $g$ is $C^{m}, g \geq 0$ and $g \leq f$ on $\left[\sigma_{k}, \tau_{k}\right]$. Since $f \leq 6 \varepsilon$ on $\left[\sigma_{k} \cdot \tau_{k}\right]$, it follows that $\sup _{t \in\left[\sigma_{k}, \tau_{k}\right]} \mid f(t)-$ $g(t) \mid \leq 6 \varepsilon$.

In conclusion, $g \in C^{m}[0,1], 0 \leq g \leq f$, and $\|f-g\|_{\infty} \leq 6 \varepsilon$.

(e) We show that for every $f \in C[0,1]^{+}$and $\varepsilon>0$ there exists a $g \in C^{m}[0,1]$ such that $0 \leq g \leq f$ and $\|g-f\|_{\infty} \leq 3 \varepsilon$. Due to (d), it only remains to deal with the 
case where $f(0)=0$ or $f(1)=0$. If $f \leq 3 \varepsilon$ on $[0,1]$, then we can take $g=0$, so we may assume that there exists $t \in[0,1]$ with $f(t)>3 \varepsilon$. We first consider the case where $f(0)=0$ and $f(1)>0$. Without loss of generality we assume that $f(1)>2 \varepsilon$. Define

$$
\begin{aligned}
\tau & :=\inf \{t \in[0,1]: f(t)>3 \varepsilon\} \text { and } \\
\sigma & :=\sup \{t \in[0, \tau]: f(t)<2 \varepsilon\} .
\end{aligned}
$$

Observe that $0<\sigma<\tau, f \geq 2 \varepsilon$ on $[\sigma, \tau]$, and $f(\tau)=3 \varepsilon$. Then, according to (d), there exists a $C^{m}$ function $g$ on $[\tau, 1]$ such that $0 \leq g \leq(f-2 \varepsilon)^{+}$and $\left\|(f-2 \varepsilon)^{+}-g\right\|_{\infty}<\varepsilon$. We take $g=0$ on $[0, \sigma]$ and with the aid of (c) we choose $g$ on $(\sigma, \tau)$ such that $g$ is a $C^{m}$ function on $[0,1]$ and such that for every $t \in(\sigma, \tau)$ we have $0 \leq g(t) \leq g(\tau)+\varepsilon$. Then, for every $t \in(\sigma, \tau)$ we have

$$
g(t) \leq g(\tau)+\varepsilon \leq(f(\tau)-2 \varepsilon)^{+}+\varepsilon \leq 2 \varepsilon \leq f(t)
$$

Thus we have constructed a $g \in C^{m}[0,1]$ such that $0 \leq g \leq f$ with $\|f-g\|_{\infty}<\leq 3 \varepsilon$.

The cases where $f(0)=0$ and $f(1)=0$, or $f(0)>0$ and $f(1)=0$ can be dealt with in a similar fashion.

(f) Let $f \in C[0,1]^{+}$. We construct a sequence $\left(f_{n}\right)_{n}$ as announced above. By means of (e), we choose $g_{1} \in C^{m}[0,1]$ with $0 \leq g_{1} \leq f$ and $\left\|f-g_{1}\right\|_{\infty}<2^{-1}$. Inductively, for $n \in \mathbb{N}$ we choose $g_{n+1} \in C^{m}[0,1]$ with $0 \leq g_{n+1} \leq f-\sum_{j=1}^{n} g_{j}$ and $\left\|\left(f-\sum_{j=1}^{n} g_{j}\right)-g_{n+1}\right\|_{\infty}<2^{-(n+1)}$. Let

$$
f_{n}:=\sum_{j=1}^{n} g_{j}, n \in \mathbb{N} .
$$

Then, as every $g_{j}$ is positive, $\left(f_{n}\right)_{n}$ is an increasing sequence in $C^{m}[0,1]^{+}$. Further, since $g_{n+1} \leq f-f_{n}$, we have $f_{n} \leq f-g_{n+1} \leq f$. Moreover, $\left\|f-f_{n+1}\right\|_{\infty}<$ $2^{-(n+1)} \rightarrow 0$ as $n \rightarrow \infty$. Finally, since $f_{n} \uparrow$ and $\left\|f-f_{n}\right\|_{\infty} \rightarrow 0$, it follows that $f=\sup _{n} f_{n}$ in $C[0,1]$.

Let us now consider extension of disjointness preserving operators on $C^{m}[0,1]$. If $T: C^{m}[0,1] \rightarrow C^{m}[0,1]$ is an injective positive and disjointness preserving operator which is continuous with respect to the norm $\|\cdot\|_{\infty}$, then there exists $\widehat{T}: C[0,1] \rightarrow$ $C[0,1]$ which is injective, positive, disjointness preserving, and such that $\left.\widehat{T}\right|_{C^{m}[0,1]}=$ $T$. Indeed, $C[0,1]$ is the uniform completion of the Riesz completion of $C^{m}[0,1]$, see [10, Corollary 2.5.10]. Thus, we can apply Theorem 6.6 and obtain the desired extension $\widehat{T}$.

In the spirit of Theorem 6.1 and Remark 6.3, we have the following consequence of Example 6.9. If $T: C[0,1] \rightarrow C[0,1]$ is an order continuous operator and its restriction to $C^{m}[0,1]$ is disjointness preserving, then $T$ is disjointness preserving.

In Theorem 6.2, it is required that for every $x \in\left(X^{\rho}\right)^{+}$, there exists a sequence $\left(x_{n}\right)_{n}$ in $X$ which converges to $x$ from below, i.e. $0 \leq i\left(x_{n}\right) \leq x$ for every $n$ and $\left\|i\left(x_{n}\right)-x\right\|_{X^{\rho}} \rightarrow 0$. In general, however, this condition is not always satisfied. For 
example, let $X=\operatorname{Pol}[0,1]$, then $X^{\rho}$ is the subspace of $C[0,1]$ consisting of piecewise polynomial functions. It is easy to find a positive $x \in X^{\rho} \backslash\{0\}$ which vanishes on a subinterval of $[0,1]$ and then there is no sequence in $X^{+}$that converges to $x$ from below.

We note that not every disjointness preserving operator on a pre-Riesz space can be extended to its Riesz completion without some strong conditions and keep the property of being disjointness preserving. For example, if $X=\operatorname{Pol}[0,1]$ the operator $T$ defined by $(T x)(t)=\int_{0}^{t} x(s) d s, t \in[0,1]$, is not disjointness preserving on $X^{\rho}$ but it is disjointness preserving on $X$, since two elements $x, y \in X$ are disjoint in $X$ only if $x=0$ or $y=0$.

Next we present a way of extending a disjointness preserving operator on a preRiesz space to the Riesz completion using the Riesz decomposition property. The range space $Y$ is required to be a Dedekind complete vector lattice.

Theorem 6.10 Let $X$ be a pervasive Archimedean pre-Riesz space with the Riesz. decomposition property, let $\left(X^{\rho}, i\right)$ be its Riesz completion, and let $Y$ be a Dedekind complete vector lattice. If $T: X \rightarrow Y$ is order bounded and disjointness preserving, then there exists an order bounded and disjointness preserving operator $S: X^{\rho} \rightarrow Y$ extending $T$ in the sense that $S \circ i=T$.

Proof For a fixed $0<y \in X^{\rho}$, since $X$ is pervasive there exists some $x \in X$ with $0<i(x) \leq y$. As $X$ is Archimedean, it follows from [10, Lemma 2.8.4] that $y=$ $\sup \{i(x) \in i(X): 0<i(x) \leq y\}$. Because $X$ is majorizing in $X^{\rho}$, there exists $z \in X$ such that $y \leq i(z)$. So $i(x) \leq y \leq i(z)$ and $x \leq z$. The order boundedness of $T$ implies that $\{$ Tx: $x \in X, 0<i(x) \leq y\}$ is bounded. Thus $\sup \{$ Tx : $x \in X, 0<i(x) \leq y\}$ exists in $Y$. So one can define a map $\widehat{T}: X^{\rho} \rightarrow Y$ via the formula

$$
\widehat{T} y=\sup \{T x: \quad x \in X, 0 \leq i(x) \leq|y|\}, y \in X^{\rho} .
$$

Obviously, this map $\widehat{T}$ is order bounded in the sense that it maps order bounded sets to order bounded sets. Moreover, $\widehat{T}$ is positively homogeneous. For $y_{1}, y_{2} \in X^{\rho}$ and $x \in X$ with $0 \leq i(x) \leq\left|y_{1}+y_{2}\right| \leq\left|y_{1}\right|+\left|y_{2}\right|$, because $X$ has the Riesz decomposition property, there exist $x_{1}, x_{2} \in X$ with $x_{1}+x_{2}=x, 0 \leq i\left(x_{1}\right) \leq\left|y_{1}\right|$ and $0 \leq i\left(x_{2}\right) \leq\left|y_{2}\right|$. Thus, $\widehat{T}\left(y_{1}+y_{2}\right)=\sup \left\{T x: x \in X, 0 \leq i(x) \leq\left|y_{1}+y_{2}\right|\right\} \leq$ $\sup \left\{T\left(x_{1}+x_{2}\right): x_{1}, x_{2} \in X, 0 \leq i\left(x_{1}\right) \leq\left|y_{1}\right|, 0 \leq i\left(x_{2}\right) \leq\left|\overline{y_{2}}\right|\right\} \leq \widehat{T} y_{1}+\widehat{T} y_{2}$. So $\widehat{T}$ is sublinear. It is clear that $T(x) \leq \widehat{T}(x)$ holds for all $x \in X$. Since $Y$ is Dedekind complete, by the Hahn-Banach extension theorem, the operator $T \circ i^{-1}: i(X) \rightarrow Y$ has a linear extension $S: X^{\rho} \rightarrow Y$ satisfying $S(u) \leq \widehat{T}(u)$ for all $u \in X^{\rho}$.

An easy argument shows that $S$ is also order bounded. It only remains to prove that $S$ is disjointness preserving. Let $y_{1}, y_{2} \in X^{\rho}$ with $y_{1} \perp y_{2}$. So $\left|y_{1}\right| \perp\left|y_{2}\right|$. By the above discussion, there exists $0 \leq x_{j} \in X$ such that $0 \leq i\left(x_{j}\right) \leq\left|y_{j}\right|$, $j=1$, 2. Hence, $i\left(x_{1}\right) \perp i\left(x_{2}\right)$ and $x_{1} \perp x_{2}$. As $T$ is disjointness preserving, we obtain $T x_{1} \perp T x_{2}$ and therefore $\widehat{T} y_{1} \perp \widehat{T} y_{2}$. Since $S\left(y_{1}\right) \leq \widehat{T}\left(y_{1}\right)$ and $-S\left(y_{1}\right)=$ $S\left(-y_{1}\right) \leq \widehat{T}\left(-y_{1}\right)=\widehat{T}\left(y_{1}\right)$, we have $\left|S\left(y_{1}\right)\right| \leq \widehat{T}\left(y_{1}\right)$. Similarly, $\left|S\left(y_{2}\right)\right| \leq \widehat{T}\left(y_{2}\right)$. Hence, $\left|S\left(y_{1}\right)\right| \wedge\left|S\left(y_{2}\right)\right| \leq \widehat{T}\left(y_{1}\right) \wedge \widehat{T}\left(y_{2}\right)=0$. Thus $S\left(y_{1}\right) \perp S\left(y_{2}\right)$. 


\section{Condition $(\beta)$ and disjointness preserving inverses}

In this section we study a technique of Abramovich and Kitover used in [1] concerning disjointness preserving inverses. They introduce a condition $(\beta)$, which can be formulated in pre-Riesz spaces as follows.

Definition 7.1 Let $X$ and $Y$ be pre-Riesz spaces and let $T: X \rightarrow Y$ be a linear operator. $T$ is said to satisfy condition $(\beta)$ if for every $x, y \in X$ with $\{x\}^{\text {dd }} \subseteq\{y\}^{\text {dd }}$ it follows that $\{T x\}^{\mathrm{dd}} \subseteq\{T y\}^{\mathrm{dd}}$.

The idea of condition $(\beta)$ traces back to [12]. In function spaces, $(\beta)$ means the following: if the support of a function is contained in the support of another function, then the same is true for the supports of their images. In the setting of vector lattices, it is shown in [1] that $(\beta)$ implies that $T^{-1}$ is disjointness preserving. We will establish that ( $\beta$ ) for $T$ implies $T^{-1}$ being disjointness preserving, provided $X$ and $Y$ are pre-Riesz spaces and $X$ is, in addition, fordable. We begin with a lemma that relates disjointness and principal bands.

Lemma 7.2 Let X be a pre-Riesz space and let $x, y \in X$. Then the following statements hold.

(i) If $x \perp y$ then $\{x\}^{\mathrm{dd}} \cap\{y\}^{\mathrm{dd}}=\{0\}$.

(ii) If $X$ is, in addition, fordable, then from $\{x\}^{\mathrm{dd}} \cap\{y\}^{\mathrm{dd}}=\{0\}$ it follows that $x \perp y$.

Proof (i) Assume that $x \perp y$, i.e. $y \in\{x\}^{\mathrm{d}}$. Hence, $\{y\}^{\mathrm{dd}} \subseteq\{x\}^{\mathrm{ddd}}=\{x\}^{\mathrm{d}}$, which implies

$$
\{x\}^{\mathrm{dd}} \cap\{y\}^{\mathrm{dd}} \subseteq\{x\}^{\mathrm{dd}} \cap\{x\}^{\mathrm{d}}=\{0\} .
$$

(ii) Assume that $\{x\}^{\mathrm{dd}} \cap\{y\}^{\mathrm{dd}}=\{0\}$. Let $\left(X^{\rho}, i\right)$ be the Riesz completion of $X$ and define $u:=|i(x)| \wedge|i(y)|$. Since $X$ is fordable, there is $S \subseteq X$ such that $\{u\}^{\mathrm{d}}=i(S)^{\mathrm{d}}$ in $X^{\rho}$. From Theorem 2.3 it follows that

$$
i^{-1}\left(\{u\}^{\mathrm{d}}\right)=i^{-1}\left(i(S)^{\mathrm{d}}\right)=S^{\mathrm{d}} .
$$

We show that $S^{\mathrm{dd}} \subseteq\{x\}^{\mathrm{dd}}$. Indeed, let $z \in\{x\}^{\mathrm{d}}$, then $i(z) \perp i(x)$, hence $i(z) \perp u$. Due to (4), $z \in i^{-1}\left(\{u\}^{\mathrm{d}}\right)=S^{\mathrm{d}}$. It follows that $\{x\}^{\mathrm{d}} \subseteq S^{\mathrm{d}}$, and therefore $S^{\mathrm{dd}} \subseteq$ $\{x\}^{\mathrm{dd}}$.

Analogously, one obtains $S^{\mathrm{dd}} \subseteq\{y\}^{\mathrm{dd}}$. The assumption yields $S \subseteq S^{\mathrm{dd}} \subseteq\{0\}$. Now (4) implies that $i(X) \subseteq\{u\}^{\overline{\mathrm{d}}}$, hence from Lemma 2.4 it follows that $u=0$. Consequently, $i(x) \perp i(y)$, which implies $x \perp y$.

If $X$ is not fordable, then Lemma 7.2 (ii) is not true, in general. Indeed, consider in Example 4.1 the case $n=3$ and the elements $x:=x^{(1)}$ and $y:=x^{(2)}$. Then $\{x\}^{\mathrm{dd}} \cap\{y\}^{\mathrm{dd}}=\{0\}$, but $x \not \perp y$. 
Theorem 7.3 Let $X$ and $Y$ be pre-Riesz spaces and let $T: X \rightarrow Y$ be a linear injective operator.

(i) If $X$ is, in addition, fordable and $T$ satisfies condition $(\beta)$, then $T^{-1}: T(X) \rightarrow X$ is disjointness preserving.

(ii) Let $T$ be surjective and disjointness preserving. If $T^{-1}$ is disjointness preserving then $T$ satisfies $(\beta)$.

Proof (i) Let $y_{1}, y_{2} \in T(X)$ be such that $y_{1} \perp y_{2}$ in $Y$ and let $x_{1}, x_{2} \in X$ be such that $T x_{1}=y_{1}$ and $T x_{2}=y_{2}$. Due to Lemma 7.2 (i) one obtains

$$
\left\{y_{1}\right\}^{\mathrm{dd}} \cap\left\{y_{2}\right\}^{\mathrm{dd}}=\{0\} .
$$

Let $u \in\left\{x_{1}\right\}^{\mathrm{dd}} \cap\left\{x_{2}\right\}^{\mathrm{dd}}$. From $u \in\left\{x_{1}\right\}^{\mathrm{dd}}$ it follows that $\{u\}^{\mathrm{dd}} \subseteq\left\{x_{1}\right\}^{\mathrm{dd}}$, hence property $(\beta)$ yields that $\{T u\}^{\mathrm{dd}} \subseteq\left\{T x_{1}\right\}^{\mathrm{dd}}$. Analogously, $\{T u\}^{\mathrm{dd}} \subseteq\left\{T x_{2}\right\}^{\mathrm{dd}}$, therefore

$$
\{T u\}^{\mathrm{dd}} \subseteq\left\{T x_{1}\right\}^{\mathrm{dd}} \cap\left\{T x_{2}\right\}^{\mathrm{dd}}=\left\{y_{1}\right\}^{\mathrm{dd}} \cap\left\{y_{2}\right\}^{\mathrm{dd}}=\{0\},
$$

which yields $T u=0$. As $T$ is injective it follows that $u=0$. Thus we obtain that $\left\{x_{1}\right\}^{\mathrm{dd}} \cap\left\{x_{2}\right\}^{\mathrm{dd}}=\{0\}$. Since $X$ is fordable, Lemma 7.2 (ii) yields that $x_{1} \perp x_{2}$. Consequently, $T^{-1}$ is disjointness preserving.

(ii) The line of reasoning here is similar to the proof of [1, Proposition 3.3]. Let $x_{1}, x_{2} \in X$ be such that $\left\{x_{1}\right\}^{\mathrm{dd}} \subseteq\left\{x_{2}\right\}^{\mathrm{dd}}$, and assume that $\left\{T x_{1}\right\}^{\mathrm{dd}} \nsubseteq\left\{T x_{2}\right\}^{\mathrm{dd}}$. This means $T x_{1} \notin\left\{T x_{2}\right\}^{\text {dd }}$, i.e. there is a $y \in\left\{T x_{2}\right\}^{\mathrm{d}}, y \neq 0$, such that $T x_{1} \not \perp y$. In particular, one has $y \perp T x_{2}$. Since $T$ is bijective, there is an $x \in X, x \neq 0$, such that $T x=y$. Since $T^{-1}$ is disjointness preserving, one obtains $x \perp x_{2}$. On the other hand, since $T$ is disjointness preserving, one gets $x \not \perp x_{1}$. This contradicts the assumption, since $x \in\left\{x_{2}\right\}^{\mathrm{d}} \subseteq\left\{x_{1}\right\}^{\mathrm{d}}$.

Open Access This article is distributed under the terms of the Creative Commons Attribution 4.0 International License (http://creativecommons.org/licenses/by/4.0/), which permits unrestricted use, distribution, and reproduction in any medium, provided you give appropriate credit to the original author(s) and the source, provide a link to the Creative Commons license, and indicate if changes were made.

\section{References}

1. Abramovich, Y.A., Kitover, A.K.: A characterization of operators preserving disjointness in terms of their inverse. Positivity 4, 205-212 (2000)

2. Abramovich, Y.A., Kitover, A.K.: Inverses of Disjointness Preserving Operators, Memoirs, vol. 679, American Mathematical Society (2000)

3. Aliprantis, C.D., Burkinshaw, O.: Positive Operators. Springer, Dordrecht (2006)

4. de Pagter, B.: A note on disjointness preserving operators. Proc. Am. Math. Soc. 90, 543-549 (1984)

5. Huijsmans, C.B., de Pagter, B.: Invertible disjointness preserving operators. Proc. Edinb. Math. Soc. 37(2), 125-132 (1994)

6. Huijsmans, C.B., Luxemburg, W.A.J.: Positive operators and semigroups on Banach lattices. In: Proceedings of a Caribbean Mathematics Foundation Conference, Springer, Dordrecht (1992)

7. Huijsmans, C.B., Wickstead, A.W.: The inverse of band preserving and disjointness preserving operators. Indag. Math. (N.S.) 3, 179-183 (1992) 
8. Kalauch, A., Lemmens, B., van Gaans, O.: Inverses of disjointness preserving operators in finite dimensional pre-Riesz spaces. Quaest. Math. 42(4), 423-430 (2019)

9. Kalauch, A., van Gaans, O.: Disjointness in partially ordered vector spaces. Positivity 10(3), 573-589 (2006)

10. Kalauch, A., van Gaans, O.: Pre-Riesz Spaces. Expositions in Mathematics, vol. 66. De Gruyter, Berlin (2019)

11. Meyer-Nieberg, P.: Banach Lattices. Springer, Berlin (1991)

12. Randrianantoanina, B.: Injective isometries in Orlicz spaces. In: Jarosz, K. (ed.), Function Spaces, Contemporary Mathematics, vol 232, Amer. Math. Soc., Providence, RI, pp. 269-287 (1999)

13. van Gaans, O.: Subspaces of normed Riesz spaces. Positivity 8, 143-164 (2004)

14. van Gaans, O.: An elementary example of an order bounded dual space that is not directed. Positivity 9(2), 265-267 (2005)

15. van Haandel, M.: Completions in Riesz Space Theory. PhD Thesis, University of Nijmegen (1993)

Publisher's Note Springer Nature remains neutral with regard to jurisdictional claims in published maps and institutional affiliations. 